\title{
The Spring Loaded Inverted Pendulum as the Hybrid Zero Dynamics of an Asymmetric Hopper
}

\author{
Ioannis Poulakakis and J. W. Grizzle
}

\begin{abstract}
A hybrid controller that induces provably stable running gaits on an Asymmetric Spring Loaded Inverted Pendulum (ASLIP) is developed. The controller acts on two levels. On the first level, continuous within-stride control asymptotically imposes a (virtual) holonomic constraint corresponding to a desired torso posture, and creates an invariant surface on which the two-degree-of-freedom restriction dynamics of the closed-loop system (i.e., the hybrid zero dynamics) is diffeomorphic to the center-of-mass dynamics of a Spring Loaded Inverted Pendulum (SLIP). On the second level, event-based control stabilizes the closed-loop hybrid system along a periodic orbit of the SLIP dynamics. The controller's performance is discussed through comparison with a second control law that creates a one-degreeof-freedom non-compliant hybrid zero dynamics. Both controllers induce identical steady-state behaviors (i.e. periodic solutions). Under transient conditions, however, the controller inducing a compliant hybrid zero dynamics based on the SLIP accommodates significantly larger disturbances, with less actuator effort, and without violation of the unilateral ground force constraints.
\end{abstract}

Index Terms - Legged robots, Spring Loaded Inverted Pendulum, Hybrid Zero Dynamics, dynamic running.

\section{INTRODUCTION}

$\mathbf{T}$ HE Spring Loaded Inverted Pendulum (SLIP) has been proposed as a canonical model of the center-of-mass dynamics of running animals and robots. Notwithstanding its apparent simplicity, the SLIP has been invaluable in uncovering basic principles of running in animals, [20], and in synthesizing empirical control laws for running robots, [36].

In the relevant literature, the SLIP is not conceived merely as a model that encodes running. It is construed as a model that implies specific high-level control hypotheses on how animals or robots coordinate their joints and limbs to produce the observed running behavior, [15], [20]. However, up to this point, much of the relevant research has been concentrated on the SLIP itself. The formal connection between the SLIP and more elaborate models that enjoy a more faithful correspondence to a typical locomotor's structure and morphology has not been fully investigated. In particular, it still remains unclear how stability conclusions obtained in the context of the SLIP can predict the behavior of more complete models. In this paper, rather than analyzing the much studied SLIP, we turn our attention to its implications in the control of running of more complete robot models. A framework is proposed

Manuscript submitted August 17, 2007; revised June 27, 2008. This work was supported by NSF grant ECS 0600869. Portions of this paper have previously appeared in the conference papers [34] and [35].

I. Poulakakis and J. W. Grizzle are with the Control Systems Laboratory, Department of Electrical Engineering and Computer Science, University of Michigan, Ann Arbor, MI 48109-2122, U.S.A. (phone: +1-734-936-3875; fax: +1-734-763-8041; e-mail: \{poulakas, grizzle\}@ eecs.umich.edu).

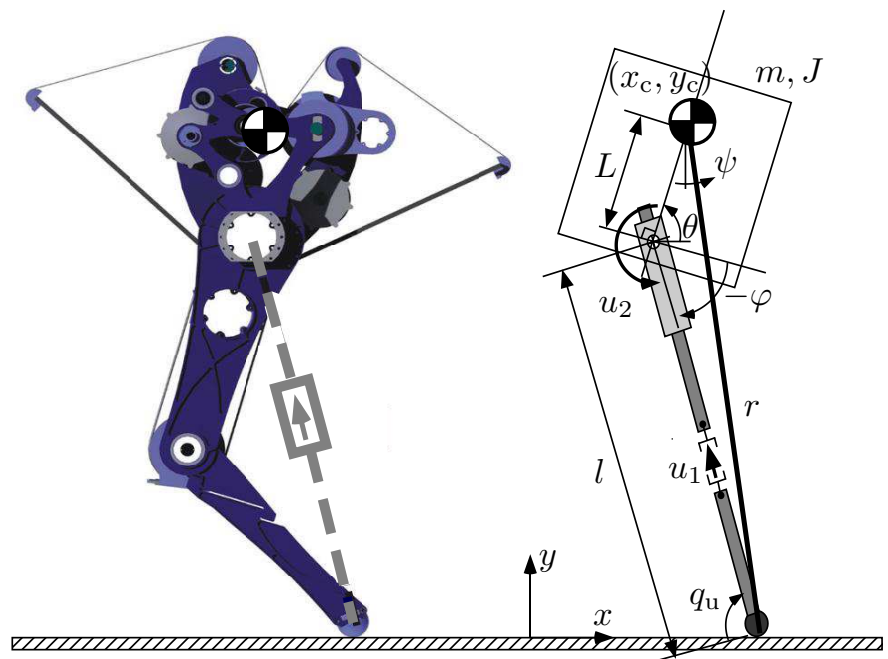

Fig. 1. Left: A mechanical drawing of a leg for a bipedal robot constructed in a collaborative effort between the University of Michigan and Carnegie Mellon University; see [21] for design principles and hardware details. The knee has a revolute series compliant actuator. Right: The Asymmetric Spring Loaded Inverted Pendulum (ASLIP). The leg force $u_{1}$ will be modeled as a spring in parallel with a prismatic force source. The ASLIP is a more faithful representation of the robot on the left than a SLIP model.

that combines established nonlinear control synthesis tools, such as the Hybrid Zero Dynamics (HZD) originally proposed in [48], with controllers obtained in the context of the SLIP e.g. [36], to induce exponentially stable running motions in a hopping model termed the Asymmetric Spring Loaded Inverted Pendulum (ASLIP); see Fig. 1. Aiming to reflect a broader purpose, the ASLIP includes torso pitch dynamics nontrivially coupled to the leg motion, an issue not addressed in the widely studied SLIP. Despite its importance, to the best of the authors' knowledge, no formal studies of the ASLIP exist. Proposing and rigorously analyzing control laws for the stabilization of the ASLIP that take advantage of SLIP controllers constitutes the primary goal of this work.

A second aspect addressed in this paper regards the performance benefits of embedding the SLIP as the hybrid zero dynamics of the ASLIP. A SLIP-embedding control law is compared with a controller that achieves a one degree-offreedom (DOF), non-compliant hybrid zero dynamics. The two controllers induce identical steady-state behaviors. Under transient conditions, however, the underlying compliant nature of the SLIP allows significantly larger disturbances to be accommodated, with less actuator effort, and without violation of the unilateral constraints between the toe and the ground.

The results presented in this paper provide the first step toward a general framework for the design of control laws 
that induce elegant, provably stable, running motions in legged robots, by combining the practical advantages of the compliant SLIP with the analytical tractability offered by the hybrid zero dynamics method.

\section{BACKGROUND}

The combined difficulties of hybrid dynamics and underactuation inherent in legged robots with point feet stymied the direct application of nonlinear controller synthesis tools, such as those in [25], to induce provably stable running motions in such robots. Instead, many empirical control procedures have been employed over the past twenty years to control hopping and running robots or robot models; see [36], [1], [17], [14], [30], [24], [10], [2] for examples of one-legged robots. In many cases, e.g. [1], [17], [2], these control procedures are inspired by Raibert's original three-part controller, regulating forward speed during flight by positioning the legs at a proper touchdown angle, and hopping height and body attitude during stance by employing leg force and hip torque; see [36]. A different class of controllers is introduced in [14]. These controllers apply impulsive (or, equivalently piecewise constant) feedback inputs at discrete time instants throughout a stride to stabilize unforced periodic solutions of a simplified model, and were found to perform well on an exact model of the hopper. The reliance of the control laws in [14] on a simplified model is removed in [24]. From a minimalist perspective, a realistic one-legged hopper is controlled using only a hip actuator in [10]. All the control laws mentioned so far incorporate sensory feedback to stabilize periodic running motions. However, as indicated in [30], stable running can be achieved using purely feed-forward periodic commands to the hip and leg motors.

The complexity of the dynamics of one-legged hoppers precluded analytically tractable stability studies, and led to introducing various simplifications: point-mass body, massless leg, zero gravity in stance, to name a few. In one of the earliest analytical works, Koditschek and Buehler explain the robust behavior of Raibert's vertical hopping controller by concentrating on the vertical oscillation of a simplified hopper; see [27]. This analysis is extended in [46] by considering the bifurcation diagram of the system's return map. Forward dynamics is added to the vertical hopper in [29] with the purpose of investigating its effect on the vertical motion. The problem of controlling forward velocity alone is examined in [13] and [40], where no control is available at the leg.

The sagittal plane model in [13] and [40] is comprised of a point-mass body attached to a massless springy leg, and is conservative with the touchdown angle being the sole control input. It corresponds to the most common configuration of the SLIP, which has appeared widely in the locomotion literature; see [15], [20] and references therein. Recently, it was discovered in [42], and, independently, in [16], that the SLIP possesses "self-stable" running gaits, though the basins of attraction may be impractically small. Control laws have been proposed that enlarge the basin of attraction of these gaits in [43], while in [3] a theoretical framework suitable for analyzing various leg placement control policies for the SLIP is developed. Three-dimensional extensions of the SLIP are also available, [41]. These research efforts produced a large variety of controllers for inducing elegant running motions in the SLIP, which exhibit very appealing properties such as large domains of attraction and minimal control effort.

A quite different paradigm for control law design combining analytical tractability with realistic models has been followed in [19], [48], and [11]; see also [47] for an integrative perspective. There, geometric nonlinear control methods have been developed that deal directly with the underactuation and hybrid dynamics present in legged robots, and induce provably asymptotically stable dynamic walking and running motions in bipedal robots. In particular, it has been shown that planar walking and running gaits can be "embedded" in the dynamics of a biped by defining a set of holonomic output functions with the control objective being to drive these outputs to zero; see [19], [48]. In essence, this method asymptotically restricts the dynamics of the closed-loop hybrid model to a lowerdimensional attractive and invariant subset of the state space. The stable periodic solutions of the dynamics restricted on this subset, called the Hybrid Zero Dynamics (HZD), encode the desired task (walking or running).

The general idea of task encoding through the enforcement of a lower-dimensional target dynamics, rather than through the prescription of a set of reference trajectories, has been employed in the control of dynamically dexterous machines, including juggling, brachiating and running robots, by Koditschek and his collaborators; see [9], [33] and [37]. The same general idea, albeit in a fully actuated setting, has been employed in [5] and [4], where the method of controlled symmetries introduced in [45] together with a generalization of Routhian reduction for hybrid systems were combined to extend passive dynamic walking gaits, such as those obtained by McGeer's passive walker [28], in three-dimensions.

Task encoding through imposing pre-specified target dynamics leaves one with the question of selecting a suitable candidate dynamical system for the targeted running behavior. On one hand, a growing body of evidence in biomechanics indicates that diverse species, when they run, tune their neural and musculoskeletal systems so that their COM bounces along as if it was following the dynamics of a SLIP; see [6], [7], [15]. On the other hand, careful consideration of the SLIP gave insight into synthesizing empirical control laws capable of stabilizing running robots with one, two and four legs, as was demonstrated in [36]. In the light of this evidence, the SLIP is construed as a dynamic model of the observed running behavior, and thus can be used as the target dynamics for legged robots; see [15] and [20].

Up to this point, however, much of this research has been concentrated on the SLIP itself, and, as was indicated in [10], controllers specifically derived for the SLIP will have to be modified in order to be successful in inducing stable running in more complete models that include pitch dynamics or energy losses. Only preliminary results in this direction are available, including [38] and [37], in which controllers for running exploit results known for the SLIP. Furthermore, the majority of control laws suitable for one-legged robot models exhibiting pitch dynamics are derived based on the 
assumption that the torso COM coincides with the hip joint; for example, see [1], [14], [30], [24], [10], [2]. The purpose of this assumption, which is crucial for the success of the control laws, is that it results in trivial coupling between the torso and leg dynamics. To the best of the authors' knowledge, only [22] and [23] addressed the asymmetric case, but stability conclusions were drawn from numerical studies only.

These observations set the stage of this research, which aims at establishing a more formal connection between the SLIP as a control target for running and more complete plant models of legged robots that include nontrivial pitch dynamics.

\section{The Asymmetric SPRING LOADED INVERTED PENDULUM}

A schematic for the Asymmetric Spring Loaded Inverted Pendulum (ASLIP) is presented in Fig. 1. The hip joint (point at which the leg is attached to the torso) does not coincide with the center of mass (COM) of the torso, which is modeled as a rigid body with mass $m$ and moment of inertia $J$ about the COM. The leg is assumed to be massless. The contact of the leg with the ground is modeled as an unactuated pin joint. The ASLIP is controlled by two inputs: a force $u_{1}$ acting along the leg, and a torque $u_{2}$ applied at the hip. In Section IX, the leg force $u_{1}$ will be modeled as a spring in parallel with a prismatic force source. In what follows, the subscripts "f $f$ " and "s" denote "flight" and "stance," respectively.

\section{A. Flight Dynamics}

The flight phase dynamics corresponds to a planar rigid body undergoing ballistic motion in a gravitational field. The configuration space $Q_{\mathrm{f}}$ of the flight phase is a simplyconnected open subset of $\mathbb{R}^{2} \times \mathbb{S}^{1}$ corresponding to physically reasonable configurations of the ASLIP, and it can be parameterized by the Cartesian coordinates $x_{\mathrm{c}}$ and $y_{\mathrm{c}}$ of the COM together with the pitch angle $\theta$, i.e. $q_{\mathrm{f}}:=\left(x_{\mathrm{c}}, y_{\mathrm{c}}, \theta\right)^{\prime} \in Q_{\mathrm{f}}$; see Fig. 1. The flight dynamics of the ASLIP can then be described by the second-order system

$$
D_{\mathrm{f}} \ddot{q}_{\mathrm{f}}+G_{\mathrm{f}}=0
$$

where $D_{\mathrm{f}}=\operatorname{diag}(m, m, J)$ and $G_{\mathrm{f}}=(0, m g, 0)^{\prime}$, with $g$ being the gravitational acceleration. The system (1) can easily be written in state-space form as

$$
\dot{x}_{\mathrm{f}}:=\frac{d}{d t}\left(\begin{array}{c}
q_{\mathrm{f}} \\
\dot{q}_{\mathrm{f}}
\end{array}\right)=\left(\begin{array}{c}
\dot{q}_{\mathrm{f}} \\
-D_{\mathrm{f}}^{-1} G_{\mathrm{f}}
\end{array}\right)=: f_{\mathrm{f}}\left(x_{\mathrm{f}}\right),
$$

evolving in $T Q_{\mathrm{f}}:=\left\{x_{\mathrm{f}}=\left(q_{\mathrm{f}}^{\prime}, \dot{q}_{\mathrm{f}}{ }^{\prime}\right)^{\prime} \mid q_{\mathrm{f}} \in Q_{\mathrm{f}}, \dot{q}_{\mathrm{f}} \in \mathbb{R}^{3}\right\}$.

The flight phase terminates when the vertical distance of the toe from the ground becomes zero. To realize this condition, the flight state vector is augmented with $\alpha_{\mathrm{f}}:=\left(l^{\mathrm{td}}, \varphi^{\mathrm{td}}\right)^{\prime} \in \mathcal{A}_{\mathrm{f}}$ an open subset of $\mathbb{R} \times \mathbb{S}^{1}$, where $l^{\text {td }}$ and $\varphi^{\text {td }}$ are the leg length and angle at touchdown, respectively, and $\dot{\alpha}_{\mathrm{f}}=0$. This means that, during flight, the leg is assumed to obtain the desired length and orientation instantaneously ${ }^{1}$, without affecting the

\footnotetext{
${ }^{1}$ Instantaneous positioning of the leg during the flight phase is only one possible foot placement strategy. Other possibilities include the case where appropriately selected functions govern the evolution of the leg variables (length and angle) in time. Such alternatives do not not have any effect on the analysis of the following sections, because the motion of the leg does not affect the second-order dynamics of the body in the flight phase.
}

motion of the torso. The threshold function $H_{\mathrm{f} \rightarrow \mathrm{s}}: T Q_{\mathrm{f}} \times$ $\mathcal{A}_{\mathrm{f}} \rightarrow \mathbb{R}$ given by

$$
H_{\mathrm{f} \rightarrow \mathrm{s}}\left(x_{\mathrm{f}}, \alpha_{\mathrm{f}}\right):=y_{\mathrm{c}}-l^{\mathrm{td}} \cos \left(\varphi^{\mathrm{td}}+\theta\right)-L \sin \theta,
$$

signifies the touchdown event at its zero crossing, and defines a smooth switching manifold $\mathcal{S}_{\mathrm{f} \rightarrow \mathrm{s}}$ in the augmented state space $\mathcal{X}_{\mathrm{f}}:=T Q_{\mathrm{f}} \times \mathcal{A}_{\mathrm{f}}$, given by

$$
\mathcal{S}_{\mathrm{f} \rightarrow \mathrm{s}}:=\left\{\left(x_{\mathrm{f}}, \alpha_{\mathrm{f}}\right) \in \mathcal{X}_{\mathrm{f}} \mid H_{\mathrm{f} \rightarrow \mathrm{s}}\left(x_{\mathrm{f}}, \alpha_{\mathrm{f}}\right)=0\right\} .
$$

Note that in (3) and (4), the parameter $\alpha_{\mathrm{f}}$ is available for control, and will eventually be chosen according to an eventbased feedback law.

\section{B. Stance Dynamics}

The configuration space $Q_{\mathrm{s}}$ of the stance phase is a simplyconnected open subset of $\mathbb{R} \times \mathbb{S}^{2}$ corresponding to physically reasonable configurations of the ASLIP, and it is parameterized by the joint coordinates: leg length $l$, leg angle with respect to the torso $\varphi$, and torso orientation $\theta$, i.e. $q_{\mathrm{s}}:=(l, \varphi, \theta)^{\prime} \in Q_{\mathrm{s}}$; see Fig. 1. Using the method of Lagrange [44, p. 255], the stance dynamics of the ASLIP can be described by the secondorder system

$$
D_{\mathrm{s}}\left(q_{\mathrm{s}}\right) \ddot{q}_{\mathrm{s}}+C_{\mathrm{s}}\left(q_{\mathrm{s}}, \dot{q}_{\mathrm{s}}\right) \dot{q}_{\mathrm{s}}+G_{\mathrm{s}}\left(q_{\mathrm{s}}\right)=B_{\mathrm{s}} u,
$$

where $u:=\left(u_{1}, u_{2}\right)^{\prime} \in \mathcal{U}$ an open subset of $\mathbb{R}^{2}$, is the input vector during stance, and the matrices in (5) are given by

$$
\begin{aligned}
& D_{\mathrm{s}}\left(q_{\mathrm{s}}\right)=\left(\begin{array}{ccc}
m & 0 & m L \cos \varphi \\
0 & m l^{2} & m l(l-L \sin \varphi) \\
m L \cos \varphi & m l(l-L \sin \varphi) & J+m L^{2}+m l(l-2 L \sin \varphi)
\end{array}\right), \\
& C_{\mathrm{s}}\left(q_{\mathrm{s}}, \dot{q}_{\mathrm{s}}\right) \dot{q}_{\mathrm{s}}=\left(\begin{array}{c}
m L \sin \varphi \dot{\theta}^{2}-m l(\dot{\varphi}+\dot{\theta})^{2} \\
m L l \cos \varphi \dot{\theta}^{2}+2 m l i(\dot{\varphi}+\dot{\theta}) \\
2 m(l-L \sin \varphi) i(\dot{\varphi}+\dot{\theta})-m L l \cos \varphi \dot{\varphi}(\dot{\varphi}+2 \dot{\theta})
\end{array}\right), \\
& G_{\mathrm{S}}\left(q_{\mathrm{s}}\right)=\left(\begin{array}{c}
m g \cos (\varphi+\theta) \\
-m g l \sin (\varphi+\theta) \\
m g L \cos \theta-m g l \sin (\varphi+\theta)
\end{array}\right), \quad B_{\mathrm{s}}=\left(\begin{array}{ll}
1 & 0 \\
0 & 1 \\
0 & 0
\end{array}\right) .
\end{aligned}
$$

The model (5) can be brought into standard state-space form by defining

$$
\begin{aligned}
\dot{x}_{\mathrm{s}} & :=\frac{d}{d t}\left(\begin{array}{c}
q_{\mathrm{s}} \\
\dot{q}_{\mathrm{s}}
\end{array}\right)=\left(\begin{array}{c}
\dot{q}_{\mathrm{s}} \\
D_{\mathrm{s}}^{-1}\left(q_{\mathrm{s}}\right)\left(-C_{\mathrm{s}}\left(q_{\mathrm{s}}, \dot{q}_{\mathrm{s}}\right) \dot{q}_{\mathrm{s}}-G_{\mathrm{s}}\left(q_{\mathrm{s}}\right)+B_{\mathrm{s}} u\right)
\end{array}\right) \\
& =: f_{\mathrm{s}}\left(x_{\mathrm{s}}\right)+g_{\mathrm{s}}\left(x_{\mathrm{s}}\right) u,
\end{aligned}
$$

where $x_{\mathrm{s}} \in T Q_{\mathrm{s}}:=\left\{\left(q_{\mathrm{s}}^{\prime}, \dot{q}_{\mathrm{s}}^{\prime}\right)^{\prime} \mid q_{\mathrm{s}} \in Q_{\mathrm{s}}, \dot{q}_{\mathrm{s}} \in \mathbb{R}^{3}\right\}=: \mathcal{X}_{\mathrm{s}}$ is the state vector.

Transition from stance to flight can be initiated by causing the acceleration of the stance leg end to be positive, i.e. directed upwards, when the ground force becomes zero. As explained in [12, Section 4], if torque discontinuities are allowed $^{2}$-as they are assumed to be in this model- when to transition into the flight phase becomes a control decision. Therefore, liftoff is assumed to occur at predetermined configurations in the stance state space that correspond to

\footnotetext{
${ }^{2}$ This is a modeling issue. In practice, the torque is continuous due to actuator dynamics. It is assumed here that the actuator time constant is small enough that it need not be modeled.
} 
the distance between the leg end and the torso COM being equal to a constant $r_{0}$, which will be fixed in the control system design; see Remark 5 in Section VI. Consequently, the threshold function $H_{\mathrm{S} \rightarrow \mathrm{f}}: T Q_{s} \rightarrow \mathbb{R}$ is defined by

$$
H_{\mathrm{s} \rightarrow \mathrm{f}}\left(x_{\mathrm{s}}\right):=r_{0}-\sqrt{L^{2}+l^{2}-2 L l \sin \varphi}
$$

and its zeroing defines the stance-to-flight switching surface

$$
\mathcal{S}_{\mathrm{S} \rightarrow \mathrm{f}}:=\left\{x_{\mathrm{s}} \in \mathcal{X}_{\mathrm{s}} \mid H_{\mathrm{s} \rightarrow \mathrm{f}}\left(x_{\mathrm{s}}\right)=0\right\} .
$$

\section{Remark 1}

Equation (7) is physically meaningful since $L^{2}+l^{2}-$ $2 L l \sin \varphi \geq(L-l)^{2} \geq 0$. Moreover, if $l \neq L$ so that $L^{2}+l^{2}-2 L l \sin \varphi \neq 0$, and if $r_{0}$ is selected so that $\mathcal{S}_{\mathrm{s} \rightarrow \mathrm{f}}$ is nonempty, then $\mathcal{S}_{\mathrm{s} \rightarrow \mathrm{f}}$ is a five-dimensional $C^{1}$ embedded submanifold of $T Q_{\mathrm{s}}$. This is a result of the regular value theorem, see Theorem (5.8) of [8, p. 78], since $H_{\mathrm{s} \rightarrow \mathrm{f}}$ is $C^{1}$ and $\partial H_{\mathrm{s} \rightarrow \mathrm{f}} / \partial x_{\mathrm{s}} \neq 0$ on $H_{\mathrm{s} \rightarrow \mathrm{f}}^{-1}(\{0\})=\mathcal{S}_{\mathrm{s} \rightarrow \mathrm{f}}$. These conditions are easily met on a physical model; see for example Table I.

\section{ASLIP Hybrid Dynamics of Running}

Let $\phi_{\mathrm{f}}:[0,+\infty) \times T Q_{\mathrm{f}} \rightarrow T Q_{\mathrm{f}}$ denote the flow generated by the flight phase vector field $f_{\mathrm{f}}$ of (2). Note that the simplicity of $f_{\mathrm{f}}$ allows for explicit calculation of the flow $\phi_{\mathrm{f}}$. When the "augmented" flight flow $\left(\phi_{\mathrm{f}}, \alpha_{\mathrm{f}}\right)$ intersects $\mathcal{S}_{\mathrm{f} \rightarrow \mathrm{s}}$, transition from flight to stance occurs. Let $\Delta_{\mathrm{f} \rightarrow \mathrm{s}}: \mathcal{S}_{\mathrm{f} \rightarrow \mathrm{s}} \rightarrow \mathcal{X}_{\mathrm{s}}$ be the flight-to-stance transition map. Similarly, let $\Delta_{\mathrm{s} \rightarrow \mathrm{f}}$ : $\mathcal{S}_{\mathrm{s} \rightarrow \mathrm{f}} \rightarrow T Q_{\mathrm{f}}$ be the stance-to-flight transition map. Both $\Delta_{\mathrm{f} \rightarrow \mathrm{s}}$ and $\Delta_{\mathrm{s} \rightarrow \mathrm{f}}$ are provided in the Appendix. Then, the openloop hybrid model of the ASLIP is

$$
\begin{gathered}
\Sigma_{\mathrm{f}}:\left\{\begin{array}{c}
\mathcal{X}_{\mathrm{f}}=T Q_{\mathrm{f}} \times \mathcal{A}_{\mathrm{f}} \\
\left(\dot{x}_{\mathrm{f}}^{\prime}, \dot{\alpha}_{\mathrm{f}}^{\prime}\right)^{\prime}=\left(f_{\mathrm{f}}^{\prime}\left(x_{\mathrm{f}}\right), 0\right)^{\prime} \\
\mathcal{S}_{\mathrm{f} \rightarrow \mathrm{s}}=\left\{\left(x_{\mathrm{f}}, \alpha_{\mathrm{f}}\right) \in \mathcal{X}_{\mathrm{f}} \mid H_{\mathrm{f} \rightarrow \mathrm{s}}\left(x_{\mathrm{f}}, \alpha_{\mathrm{f}}\right)=0\right\} \\
x_{\mathrm{s}}^{+}=\Delta_{\mathrm{f} \rightarrow \mathrm{s}}\left(x_{\mathrm{f}}^{-}, \alpha_{\mathrm{f}}\right)
\end{array}\right. \\
\Sigma_{\mathrm{s}}:\left\{\begin{array}{c}
\mathcal{X}_{\mathrm{s}}=T Q_{\mathrm{s}} \\
\dot{x}_{\mathrm{s}}=f_{\mathrm{s}}\left(x_{\mathrm{s}}\right)+g_{\mathrm{s}}\left(x_{\mathrm{s}}\right) u \\
\mathcal{S}_{\mathrm{s} \rightarrow \mathrm{f}}=\left\{x_{\mathrm{s}} \in \mathcal{X}_{\mathrm{s}} \mid H_{\mathrm{s} \rightarrow \mathrm{f}}\left(x_{\mathrm{s}}\right)=0\right\} \\
x_{\mathrm{f}}^{+}=\Delta_{\mathrm{s} \rightarrow \mathrm{f}}\left(x_{\mathrm{s}}^{-}\right)
\end{array}\right.
\end{gathered}
$$

where $x_{i}^{-}=\lim _{\tau \nearrow t} x_{i}(\tau)$ and $x_{i}^{+}=\lim _{\tau \searrow t} x_{i}(\tau), i \in\{\mathrm{f}, \mathrm{s}\}$, are the left and right limits of the stance and flight solutions.

The subsystems $\Sigma_{\mathrm{f}}$ and $\Sigma_{\mathrm{s}}$ can be combined into a single system with impulse effects $\Sigma^{\text {ASLIP }}$ describing the openloop hybrid dynamics of the ASLIP; see [47, pp. 252-254], for a discussion of the related geometry. Define the time-totouchdown function $T_{\mathrm{f}}: \mathcal{X}_{\mathrm{f}} \rightarrow \mathbb{R} \cup\{\infty\}$, as

$T_{\mathrm{f}}\left(x_{\mathrm{f}, 0}, \alpha_{\mathrm{f}}\right):=\left\{\begin{aligned} \inf \left\{t \in[0,+\infty) \mid\left(\phi_{\mathrm{f}}\left(t, x_{\mathrm{f}, 0}\right), \alpha_{\mathrm{f}}\right) \in \mathcal{S}_{\mathrm{f} \rightarrow \mathrm{s}}\right\}, \\ \quad \text { if } \exists t \text { such that }\left(\phi_{\mathrm{f}}\left(t, x_{\mathrm{f}, 0}\right), \alpha_{\mathrm{f}}\right) \in \mathcal{S}_{\mathrm{f} \rightarrow \mathrm{s}} \\ \infty, \text { otherwise. }\end{aligned}\right.$

The flow map $^{3} F_{\mathrm{f}}: \mathcal{X}_{\mathrm{f}} \rightarrow \mathcal{S}_{\mathrm{f} \rightarrow \mathrm{s}}$ for the (augmented) flight phase can then be given by the rule $\left(x_{\mathrm{f}, 0}, \alpha_{\mathrm{f}}\right) \mapsto$

\footnotetext{
${ }^{3}$ The definition of the flight flow map presupposes the existence of a time instant $t$ such that $\left(\phi_{\mathrm{f}}\left(t, x_{\mathrm{f}, 0}\right), \alpha_{\mathrm{f}}\right) \in \mathcal{S}_{\mathrm{f} \rightarrow \mathrm{s}}$. The case where no such time instant exists does not correspond to periodic running motions.
}

$\left(\phi_{\mathrm{f}}\left(T_{\mathrm{f}}\left(x_{\mathrm{f}, 0}, \alpha_{\mathrm{f}}\right), x_{\mathrm{f}, 0}\right), \alpha_{\mathrm{f}}\right) \in \mathcal{S}_{\mathrm{f} \rightarrow \mathrm{s}}$. Let $\Delta: \mathcal{S}_{\mathrm{s} \rightarrow \mathrm{f}} \times \mathcal{A}_{\mathrm{f}} \rightarrow \mathcal{X}_{\mathrm{s}}$ be the map

$$
\Delta\left(x_{\mathrm{s}}^{-}, \alpha_{\mathrm{f}}\right):=\Delta_{\mathrm{f} \rightarrow \mathrm{s}}\left[F_{\mathrm{f}}\left(\Delta_{\mathrm{s} \rightarrow \mathrm{f}}\left(x_{\mathrm{s}}^{-}\right), \alpha_{\mathrm{f}}\right)\right] .
$$

The map $\Delta$ "compresses" the flight phase into an "event," and can be thought of as a (generalized) "impact map" [12], or a "reset map" [5]. In this setting, the hybrid dynamics of the ASLIP becomes

$$
\Sigma^{\operatorname{ASLIP}}:\left\{\begin{aligned}
\dot{x}_{\mathrm{s}}= & f_{\mathrm{s}}\left(x_{\mathrm{s}}\right)+g_{\mathrm{s}}\left(x_{\mathrm{s}}\right) u \\
& x_{\mathrm{s}}^{-} \notin \mathcal{S}_{\mathrm{s} \rightarrow \mathrm{f}} \\
x_{\mathrm{s}}^{+}= & \Delta\left(x_{\mathrm{s}}^{-}, \alpha_{\mathrm{f}}\right) \\
& x_{\mathrm{s}}^{-} \in \mathcal{S}_{\mathrm{s} \rightarrow \mathrm{f}}, \alpha_{\mathrm{f}} \in \mathcal{A}_{\mathrm{f}}
\end{aligned}\right.
$$

The left and right limits $x_{\mathrm{s}}^{-}$and $x_{\mathrm{s}}^{+}$correspond to the states "just prior to liftoff" and "just after touchdown," respectively. Note also that in (12), only the argument $x_{\mathrm{s}}^{-} \in \mathcal{S}_{\mathrm{s} \rightarrow \mathrm{f}}$ triggers liftoff; $\alpha_{\mathrm{f}}$ affects the initial conditions of the continuous part of (12). The system $\Sigma^{\mathrm{ASLIP}}$ has the typical form of a system with impulse effects, i.e., it is defined on a single chart $\mathcal{X}_{\mathrm{s}}$, where the states evolve, together with the map $\Delta$, which reinitializes the differential equation at liftoff.

\section{OVERVIEW OF THE CONTROL LAW}

In this section, the framework within which controllers for the ASLIP are designed is outlined. Generally speaking, for the two controllers that will be presented in this paper, the purpose of the feedback law is to coordinate the actuated degrees of freedom of the ASLIP so that a lower-dimensional hybrid system emerges from the closed-loop ASLIP dynamics; this lower-dimensional dynamical system serves as a target for the control of the ASLIP and governs its asymptotic behavior. This statement will be made mathematically precise in the following sections. In this section, only the general guidelines are briefly described. To keep the exposition concise, the equations associated with the control laws are not included here; see [35] for details.

The feedback law exploits the hybrid nature of the system by introducing control action on two levels; see Fig. 2. On the first level, a continuous-time feedback law $\Gamma_{\mathrm{c}}$ is employed in the stance phase with the purpose of creating an invariant and attractive submanifold $\mathcal{Z}$ embedded in the stance state space, on which the closed-loop dynamics have desired properties. On the second level, event-based updates of controller parameters are performed at transitions from stance to flight. Generally, the event-based parameter update law is organized in an inner/outer-loop architecture, with the innerloop controller $\Gamma_{\mathrm{s}}$ intended to render the surface $\mathcal{Z}$ invariant under the reset map. This condition is referred to as hybrid invariance, and it leads to the creation of a reduced-order hybrid subsystem called the Hybrid Zero Dynamics (HZD), which governs the stability properties of the full-order ASLIP; see [32] and [48] for details. In cases where the in-stride controller $\Gamma_{\mathrm{c}}$ achieves hybrid invariance, $\Gamma_{\mathrm{s}}$ is not needed and may be excluded from the controller design; Section VI presents one such example. Finally, the outer-loop controller $\Gamma_{\mathrm{f}}$ completes the control design by ensuring that the resulting HZD is exponentially stable. 


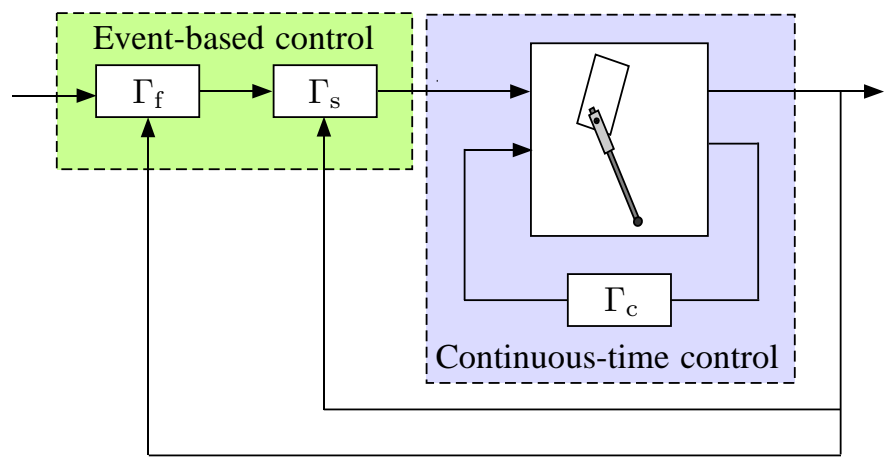

Fig. 2. Feedback diagram presenting the basic structure of the controllers.

In Sections VI and VIII we particularize these ideas through explicit constructions of two sets of feedback laws $\Gamma_{c}, \Gamma_{\mathrm{s}}$ and $\Gamma_{\mathrm{f}}$ that achieve the control objectives. In Section VI, the objective is to coordinate the actuated DOFs of the ASLIP so that the compliant SLIP emerges as the HZD; this controller is referred to as the SLIP-embedding controller. In Section VIII, the objective is to impose suitably parameterized virtual holonomic constraints on the ASLIP so that a one-DOF mechanical system arises as its HZD; because, in this case, the resulting HZD cannot be compliant, we refer to this controller as the rigid target model controller. Fundamental differences in the two control laws are highlighted in Section IX, illustrating the benefits of designing the HZD to accommodate compliance, such as in the SLIP-embedding controller.

\section{TARget Model: The EnERgy-Stabilized SLIP}

In this section, the target model for the SLIP-embedding controller is introduced. The standard SLIP consists of a point mass attached to a massless prismatic spring, and it is passive (no torque inputs) and conservative (no energy losses), thus precluding the existence of exponentially stable periodic orbits; see [3], [16]. In this paper, we consider a variant of the SLIP, where the leg force is allowed to be nonconservative. The purpose of this modification is to introduce control authority over the total energy, which is no longer conserved as in the standard SLIP, thus leading to the existence of exponentially stable periodic orbits. This system, called the Energy-Stabilized SLIP (ES-SLIP), is presented in Fig. 3.

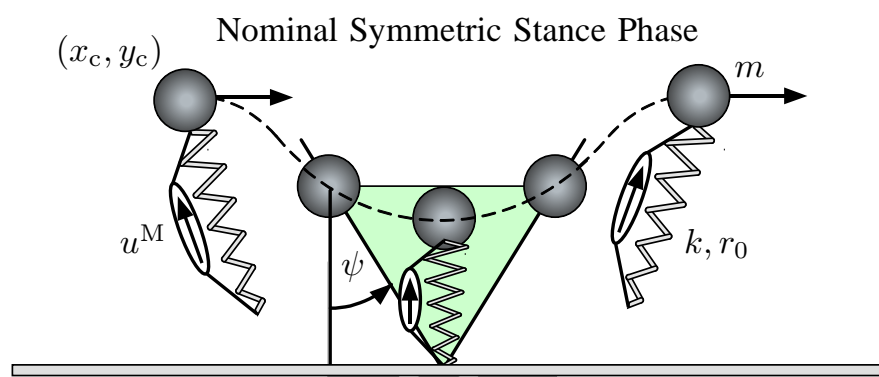

Fig. 3. The Energy-Stabilized SLIP (ES-SLIP), with a prismatic actuator (force source) in parallel with the spring.

The derivation of the hybrid model for the ES-SLIP is similar to that of the ASLIP, thus the exposition in this section will be terse. Moreover, only the closed-loop hybrid dynamics of the ES-SLIP will be presented. In what follows, the superscript " $M$ " denotes the ES-SLIP target model. The flight and stance configuration spaces $Q_{\mathrm{f}}^{\mathrm{M}}$ and $Q_{\mathrm{s}}^{\mathrm{M}}$, respectively, will both be parameterized by the Cartesian coordinates of the COM $\left(x_{\mathrm{c}}, y_{\mathrm{c}}\right) \in Q_{\mathrm{f}}^{\mathrm{M}}=Q_{\mathrm{s}}^{\mathrm{M}}=: Q^{\mathrm{M}}$ a simply-connected open subset of $\left\{\left(x_{\mathrm{c}}, y_{\mathrm{c}}\right) \in \mathbb{R}^{2} \backslash\{(0,0)\} \mid y_{\mathrm{c}}>0\right\}$. Hence, the system dynamics evolves in the state space $\mathcal{X}^{\mathrm{M}}:=T Q^{\mathrm{M}}=$ $\left\{x^{\mathrm{M}}=\operatorname{col}\left(q^{\mathrm{M}}, \dot{q}^{\mathrm{M}}\right) \mid q^{\mathrm{M}} \in Q^{\mathrm{M}}, \dot{q}^{\mathrm{M}} \in \mathbb{R}^{2}\right\}$.

In order to accommodate perturbations away from the nominal energy, the conservative force $F_{\text {el }}$ developed by the springy leg of the standard SLIP is modified to include a nonconservative feedback component $u^{\mathrm{M}}=\Gamma_{\mathrm{c}}^{\mathrm{M}}\left(x^{\mathrm{M}}\right)$. The purpose of $u^{\mathrm{M}}$ is to stabilize the total energy of the system at a desired nominal level $\bar{E}$, and is achieved by

$$
\Gamma_{\mathrm{c}}^{\mathrm{M}}\left(x^{\mathrm{M}}\right)=-K_{\mathrm{P}}^{\mathrm{E}} \frac{x_{\mathrm{c}} \dot{x}_{\mathrm{c}}+y_{\mathrm{c}} \dot{y}_{\mathrm{c}}}{\sqrt{x_{\mathrm{c}}^{2}+y_{\mathrm{c}}^{2}}}\left[E\left(x^{\mathrm{M}}\right)-\bar{E}\right],
$$

where $E\left(x^{\mathrm{M}}\right)$ is the total energy, and $K_{\mathrm{P}}^{\mathrm{E}}$ is a positive gain.

To regulate the forward speed, the following event-based control law is employed

$$
\psi=\Gamma_{\mathrm{f}}^{\mathrm{M}}\left(\left(x^{\mathrm{M}}\right)^{-}\right)=\bar{\psi}+K_{\dot{x}}\left(\dot{x}_{\mathrm{c}}^{-}-\dot{\bar{x}}_{\mathrm{c}}\right),
$$

where $\bar{\psi}$ and $\dot{\bar{x}}_{\mathrm{c}}$ specify the nominal touchdown angle and forward speed, respectively, $\dot{x}_{\mathrm{c}}^{-}$is the actual forward speed just prior to liftoff, and $K_{\dot{x}}$ is a positive gain.

\section{Remark 2}

It can be recognized that (14) corresponds to a variation of Raibert's speed controller, [36, pp. 44-47]. Feedback control laws similar to (13) and (14) exist in the literature; the particular ones used here are for illustrative purposes only. It is emphasized that many other in-stride or event-based controllers could have been used to stabilize the SLIP. For instance, energy stabilization in nonconservative monopedal models has been demonstrated using linear (leg) and rotational (hip) actuation in [2] and [10], respectively. On the other hand, a large variety of event-based controllers exist for the SLIP, e.g. [3], [36], [39], [43], which are known to have very appealing properties. In the next section, we develop rigorously a controller for the ASLIP that affords the direct use of control laws available for the SLIP.

Under the influence of the feedback laws (13) and (14), the closed-loop ES-SLIP hybrid dynamics can be obtained as

$$
\Sigma_{\mathrm{cl}}^{\mathrm{M}}:\left\{\begin{aligned}
\dot{x}^{\mathrm{M}} & =f_{\mathrm{s}, \mathrm{cl}}^{\mathrm{M}}\left(x^{\mathrm{M}}\right),\left(x^{\mathrm{M}}\right)^{-} \notin \mathcal{S}_{\mathrm{s} \rightarrow \mathrm{f}}^{\mathrm{M}} \\
\left(x^{\mathrm{M}}\right)^{+} & =\Delta_{\mathrm{cl}}^{\mathrm{M}}\left(\left(x^{\mathrm{M}}\right)^{-}\right),\left(x^{\mathrm{M}}\right)^{-} \in \mathcal{S}_{\mathrm{s} \rightarrow \mathrm{f}}^{\mathrm{M}},
\end{aligned}\right.
$$

where $f_{\mathrm{s}, \mathrm{cl}}^{\mathrm{M}}\left(x^{\mathrm{M}}\right)$ is the closed-loop stance vector field, which is given below for future use,

$$
f_{\mathrm{s}, \mathrm{cl}}^{\mathrm{M}}\left(x^{\mathrm{M}}\right)=\left(\begin{array}{c}
\dot{x}_{\mathrm{c}} \\
\dot{y}_{\mathrm{c}} \\
\frac{1}{m} \frac{x_{\mathrm{c}}}{\sqrt{x_{\mathrm{c}}^{2}+y_{\mathrm{c}}^{2}}}\left(F_{\mathrm{el}}+\Gamma_{\mathrm{c}}^{\mathrm{M}}\left(x^{\mathrm{M}}\right)\right) \\
\frac{1}{m} \frac{y_{\mathrm{c}}}{\sqrt{x_{\mathrm{c}}^{2}+y_{\mathrm{c}}^{2}}}\left(F_{\mathrm{el}}+\Gamma_{\mathrm{c}}^{\mathrm{M}}\left(x^{\mathrm{M}}\right)\right)-g
\end{array}\right) ;
$$

$F_{\text {el }}$ is the elastic force developed by the prismatic spring of the leg, which is assumed to be generated by a radial potential 
$\mathcal{V}_{\mathrm{el}}^{\mathrm{M}}\left(r\left(x_{\mathrm{c}}, y_{\mathrm{c}}\right)\right)$ with $r\left(x_{\mathrm{c}}, y_{\mathrm{c}}\right)=\sqrt{x_{\mathrm{c}}^{2}+y_{\mathrm{c}}^{2}}$ as

$$
F_{\mathrm{el}}=\left.\frac{d \mathcal{V}_{\mathrm{el}}^{\mathrm{M}}(r)}{d r}\right|_{r=\sqrt{x_{\mathrm{c}}^{2}+y_{\mathrm{c}}^{2}}} .
$$

Assuming, for definiteness, that the spring is linear,

$$
F_{\mathrm{el}}=k\left(r_{0}+\Delta r-\sqrt{x_{\mathrm{c}}^{2}+y_{\mathrm{c}}^{2}}\right)
$$

$k$ is the spring constant, $r_{0}$ the nominal leg length (determining touchdown), and $\Delta r$ a (constant) pretention; see Fig. 3.

In (15), the switching surface

$$
\mathcal{S}_{\mathrm{s} \rightarrow \mathrm{f}}^{\mathrm{M}}:=\left\{x^{\mathrm{M}} \in \mathcal{X}^{\mathrm{M}} \mid H_{\mathrm{s} \rightarrow \mathrm{f}}^{\mathrm{M}}\left(x^{\mathrm{M}}\right)=0\right\},
$$

where

$$
H_{\mathrm{s} \rightarrow \mathrm{f}}^{\mathrm{M}}\left(x^{\mathrm{M}}\right):=r_{0}-\sqrt{x_{\mathrm{c}}^{2}+y_{\mathrm{c}}^{2}},
$$

is a three-dimensional $C^{1}$ embedded submanifold of $\mathcal{X}^{\mathrm{M}}$, for reasons similar to those mentioned in Remark 1.

\section{Remark 3}

To explain (19) and (20), the liftoff condition is assumed to occur when the leg length obtains a particular value, namely $r_{0}$, as is the case for the conservative SLIP.

Finally, the closed-loop reset map $\Delta_{\mathrm{cl}}^{\mathrm{M}}: \mathcal{S}_{\mathrm{s} \rightarrow \mathrm{f}}^{\mathrm{M}} \rightarrow \mathcal{X}^{\mathrm{M}}$ in (15) is defined by ${ }^{4}$

$$
\Delta_{\mathrm{cl}}^{\mathrm{M}}:=\Delta_{\mathrm{f} \rightarrow \mathrm{s}}^{\mathrm{M}} \circ F_{\mathrm{f}}^{\mathrm{M}} \circ\left(\Delta_{\mathrm{s} \rightarrow \mathrm{f}}^{\mathrm{M}} \times \Gamma_{\mathrm{f}}^{\mathrm{M}}\right),
$$

where $\Delta_{\mathrm{s} \rightarrow \mathrm{f}}^{\mathrm{M}}: \mathcal{S}_{\mathrm{s} \rightarrow \mathrm{f}}^{\mathrm{M}} \rightarrow \mathcal{X}^{\mathrm{M}}$ and $\Delta_{\mathrm{f} \rightarrow \mathrm{s}}^{\mathrm{M}}: \mathcal{S}_{\mathrm{f} \rightarrow \mathrm{s}}^{\mathrm{M}} \rightarrow \mathcal{X}^{\mathrm{M}}$ are the ES-SLIP stance-to-flight and flight-to-stance transition maps, respectively. Due to the fact that both the flight and stance state spaces are parameterized by the same coordinates, the transition maps simply correspond to the identity map on $\mathcal{X}^{\mathrm{M}}$, i.e. $\Delta_{\mathrm{s} \rightarrow \mathrm{f}}^{\mathrm{M}}=\Delta_{\mathrm{f} \rightarrow \mathrm{s}}^{\mathrm{M}}=\mathrm{id}_{\mathcal{X}^{\mathrm{M}}}$. In $(21), F_{\mathrm{f}}^{\mathrm{M}}: \mathcal{X}^{\mathrm{M}} \times \mathcal{A}_{\mathrm{f}}^{\mathrm{M}} \rightarrow \mathcal{S}_{\mathrm{s} \rightarrow \mathrm{f}}^{\mathrm{M}}$ is the ES-SLIP flight flow map, defined analogously with the ASLIP flight flow map; $\mathcal{A}_{\mathrm{f}}^{\mathrm{M}}$ is an open subset of $\mathbb{S}^{1}$, containing physically reasonable values for the touchdown angle $\psi$.

In order to study the stability properties of periodic orbits of $\Sigma_{\mathrm{cl}}^{\mathrm{M}}$, the method of Poincaré is used. The Poincaré section is selected to be the surface $\mathcal{S}_{\mathrm{s} \rightarrow \mathrm{f}}^{\mathrm{M}}$ defined by (19). Let $\phi_{\mathrm{s}, \mathrm{cl}}^{\mathrm{M}}$ : $[0,+\infty) \times \mathcal{X}^{\mathrm{M}} \rightarrow \mathcal{X}^{\mathrm{M}}$ be the flow generated by $f_{\mathrm{s}, \mathrm{cl}}^{\mathrm{M}}$, and define the time-to-liftoff function $T_{\mathrm{s}}^{\mathrm{M}}: \mathcal{X}^{\mathrm{M}} \rightarrow \mathbb{R} \cup\{\infty\}$, in a similar fashion as (10), by

$T_{\mathrm{s}}^{\mathrm{M}}\left(x_{0}^{\mathrm{M}}\right):=\left\{\begin{array}{c}\inf \left\{t \in[0,+\infty) \mid \phi_{\mathrm{s}, \mathrm{cl}}^{\mathrm{M}}\left(t, x_{0}^{\mathrm{M}}\right) \in \mathcal{S}_{\mathrm{s} \rightarrow \mathrm{f}}^{\mathrm{M}}\right\}, \\ \quad \text { if } \exists t \text { such that } \phi_{\mathrm{s}, \mathrm{cl}}^{\mathrm{M}}\left(t, x_{0}^{\mathrm{M}}\right), \in \mathcal{S}_{\mathrm{s} \rightarrow \mathrm{f}}^{\mathrm{M}} \\ \infty, \text { otherwise. }\end{array}\right.$

Then, the Poincaré map $\mathcal{P}^{\mathrm{M}}: \mathcal{S}_{\mathrm{s} \rightarrow \mathrm{f}}^{\mathrm{M}} \rightarrow \mathcal{S}_{\mathrm{s} \rightarrow \mathrm{f}}^{\mathrm{M}}$ is defined by

$$
\mathcal{P}^{\mathrm{M}}:=\phi_{\mathrm{s}, \mathrm{cl}}^{\mathrm{M}} \circ\left[\left(T_{\mathrm{s}}^{\mathrm{M}} \circ \Delta_{\mathrm{cl}}^{\mathrm{M}}\right) \times \Delta_{\mathrm{cl}}^{\mathrm{M}}\right] \text {. }
$$

${ }^{4}$ Notation: Let $f_{1}: \mathcal{X} \rightarrow \mathcal{Y}_{1}$ and $f_{2}: \mathcal{X} \rightarrow \mathcal{Y}_{2}$, and define $f_{1} \times f_{2}:$ $\mathcal{X} \rightarrow \mathcal{Y}_{1} \times \mathcal{Y}_{2}$ by $\left(f_{1} \times f_{2}\right)(x)=\left(f_{1}(x), f_{2}(x)\right), x \in \mathcal{X}$.

\section{Main Result: The SLIP-Embedding Controller}

As was mentioned in Section IV, the control action takes place on two hierarchical levels. On the first level, continuous in-stride control is exerted during the stance phase to stabilize the torso at a desired posture, and to create an invariant manifold on which the ES-SLIP dynamics can be imposed. On the second level, an event-based SLIP controller is used to stabilize a periodic orbit of the system. These results are summarized in the following theorem and corollary.

\section{Theorem 1 (SLIP-embedding controller)}

Let $\hat{Q}_{\mathrm{s}}:=\left\{q_{\mathrm{s}} \in Q_{\mathrm{s}} \mid l \neq L \sin \varphi\right\}$. Then, for every $\epsilon>$ 0 , there exists a $C^{1}$ in-stride (continuous) control law $u=$ $\Gamma_{\mathrm{c}}^{\epsilon}\left(x_{\mathrm{s}}\right)$, and a $C^{1}$ event-based (discrete) control law $\alpha_{\mathrm{f}}=$ $\Gamma_{\mathrm{f}}\left(x_{\mathrm{s}}^{-}\right)$such that the following hold:

A. In-stride Continuous Control

There exists a map $\Phi: T \hat{Q}_{\mathrm{s}} \rightarrow \mathbb{R}^{6}$ that is a diffeomorphism onto its image, and such that, in coordinates $x=\left(\eta^{\prime}, z^{\prime}\right)^{\prime}:=$ $\Phi\left(x_{\mathrm{s}}\right) \in \mathbb{R}^{6}$, the closed-loop model

$$
f_{\mathrm{s}, \mathrm{cl}}^{\epsilon}\left(x_{\mathrm{s}}\right):=f_{\mathrm{s}}\left(x_{\mathrm{s}}\right)+g_{\mathrm{s}}\left(x_{\mathrm{s}}\right) \Gamma_{\mathrm{c}}^{\epsilon}\left(x_{\mathrm{s}}\right)
$$

satisfies:

A.1) the vector field ${ }^{5}$

$$
\tilde{f}_{\mathrm{s}, \mathrm{cl}}^{\epsilon}(x):=\left.\left(\frac{\partial \Phi}{\partial x_{\mathrm{s}}} f_{\mathrm{s}, \mathrm{cl}}^{\epsilon}\left(x_{\mathrm{s}}\right)\right)\right|_{x_{\mathrm{s}}=\Phi^{-1}(x)}
$$

has the form

$$
\tilde{f}_{\mathrm{s}, \mathrm{cl}}^{\epsilon}(x)=\left(\begin{array}{c}
\tilde{f}_{\mathrm{s}, \mathrm{cl}, 1: 2}^{\epsilon}(\eta) \\
\tilde{f}_{\mathrm{s}, \mathrm{cl}, 3: 6}(\eta, z)
\end{array}\right)
$$

A.2) the set $\mathcal{Z}:=\left\{x \in \mathbb{R}^{6} \mid \eta=0\right\}$ is a smooth fourdimensional $C^{1}$ embedded submanifold of $\mathbb{R}^{6}$ that is invariant under the stance flow, i.e. $x \in \mathcal{Z}$ implies $\tilde{f}_{\mathrm{s}, \mathrm{cl}}^{\epsilon}(x) \in T_{x} \mathcal{Z}$, and the set $\mathcal{S}_{\mathrm{s} \rightarrow \mathrm{f}} \cap \mathcal{Z}$, where $\mathcal{S}_{\mathrm{s} \rightarrow \mathrm{f}}$ is given by (8), is a co-dimension one $C^{1}$ submanifold of $\mathcal{Z}$;

A.3) the transverse dynamics $\tilde{f}_{\mathrm{s}, \mathrm{cl}, 1: 2}^{\epsilon}(\eta)$ takes the form

$$
\tilde{f}_{\mathrm{s}, \mathrm{cl}, 1: 2}^{\epsilon}(\eta)=A(\epsilon) \eta,
$$

and it exponentially contracts as $\epsilon \rightarrow 0$, i.e. $\lim _{\epsilon \backslash 0} e^{A(\epsilon)}=0$; A.4) the restriction dynamics

$$
\left.\tilde{f}_{\mathrm{s}, \mathrm{cl}}^{\epsilon}(x)\right|_{\mathcal{Z}}=\tilde{f}_{\mathrm{s}, \mathrm{cl}, 3: 6}(0, z)
$$

is diffeomorphic to the ES-SLIP stance phase closed-loop dynamics $f_{\mathrm{s}, \mathrm{cl}}^{\mathrm{M}}$ given by (16).

B. Event-based Discrete Control

The closed-loop reset map $\Delta_{\mathrm{cl}}: \mathcal{S}_{\mathrm{s} \rightarrow \mathrm{f}} \rightarrow T \hat{Q}_{\mathrm{s}}$ defined by

$$
\Delta_{\mathrm{cl}}=\Delta_{\mathrm{f} \rightarrow \mathrm{s}} \circ F_{\mathrm{f}} \circ\left(\Delta_{\mathrm{s} \rightarrow \mathrm{f}} \times \Gamma_{\mathrm{f}}\right) \text {, }
$$

where the maps $\Delta_{\mathrm{f} \rightarrow \mathrm{s}}, \Delta_{\mathrm{s} \rightarrow \mathrm{f}}$ and $F_{\mathrm{f}}$ have been defined in Section III-C, satisfies

B.1) $\Delta_{\mathrm{cl}}\left(\mathcal{S}_{\mathrm{s} \rightarrow \mathrm{f}} \cap \mathcal{Z}\right) \subset \mathcal{Z}$, i.e. $\mathcal{S}_{\mathrm{s} \rightarrow \mathrm{f}} \cap \mathcal{Z}$ is hybrid invariant;

B.2) the restricted reset map $\left.\Delta_{\mathrm{cl}}\right|_{\mathcal{Z}}$ is diffeomorphic to the ES-SLIP closed-loop reset map $\Delta_{\mathrm{cl}}^{\mathrm{M}}$ defined by (21).

\footnotetext{
${ }^{5}$ Notation: Different symbols are used to denote the representations of vector fields and functions in different coordinates. Such distinction is not made for surfaces since the corresponding coordinates are clear from the context.
} 
For $\epsilon>0$ a given constant, the closed-loop hybrid dynamics of the ASLIP under the continuous and event-based feedback control laws of Theorem 1 takes the form

$$
\Sigma_{\mathrm{cl}}^{\mathrm{ASLIP}}:\left\{\begin{aligned}
\dot{x} & =\tilde{f}_{\mathrm{s}, \mathrm{cl}}^{\epsilon}(x), x^{-} \notin \mathcal{S}_{\mathrm{s} \rightarrow \mathrm{f}} \\
x^{+} & =\tilde{\Delta}_{\mathrm{cl}}\left(x^{-}\right), x^{-} \in \mathcal{S}_{\mathrm{s} \rightarrow \mathrm{f}},
\end{aligned}\right.
$$

where $\mathcal{S}_{\mathrm{S} \rightarrow \mathrm{f}}$ was defined in (8), and $\tilde{\Delta}_{\mathrm{cl}}:=\Phi \circ \Delta_{\mathrm{cl}} \circ \Phi^{-1}$ is the representation of the closed-loop reset map in the $x$ coordinates. The stability properties of $\Sigma_{\mathrm{cl}}^{\mathrm{ASLIP}}$ will be studied via the corresponding Poincaré return map $\mathcal{P}^{\epsilon}: \mathcal{S}_{\mathrm{s} \rightarrow \mathrm{f}} \rightarrow \mathcal{S}_{\mathrm{s} \rightarrow \mathrm{f}}$, which is defined analogously to $\mathcal{P}^{\mathrm{M}}$ of Section V; see (23). As is described in detail in [31], the structure imposed on the ASLIP by the feedback laws of Theorem 1 results in the map $\left.\mathcal{P}^{\epsilon}\right|_{\mathcal{Z}}: \mathcal{S}_{\mathrm{s} \rightarrow \mathrm{f}} \cap \mathcal{Z} \rightarrow \mathcal{S}_{\mathrm{s} \rightarrow \mathrm{f}} \cap \mathcal{Z}$ being independent of $\epsilon$ and $\left.\mathcal{P}^{\epsilon}\right|_{\mathcal{Z}} \cong \mathcal{P}^{\mathrm{M}}$, i.e. the restricted Poincaré map is well defined and is diffeomorphic to the ES-SLIP Poincare map. The following corollary is an immediate consequence of Theorem 1 in view of the results in [31].

Corollary 1 (Exponential Stability of $\Sigma_{\mathrm{cl}}^{\mathrm{ASLIP}}$ )

Let $\left(x^{\mathrm{M}}\right)^{*}$ be a fixed point of $\mathcal{P}^{\mathrm{M}}$ and $x^{*}$ a fixed point of $\mathcal{P}^{\epsilon}$. There exist $\bar{\epsilon}>0$ such that, for all $\epsilon \in(0, \bar{\epsilon}), x^{*}$ is exponentially stable, if, and only if, $\left(x^{\mathrm{M}}\right)^{*}$ is exponentially stable.

Before continuing with the proof of Theorem 1, which will be given in Section VII, a few remarks are in order.

\section{Remark 4}

The conditions $l \neq L$ of Remark 1 and $l \neq L \sin \varphi$ of Theorem 1 are both satisfied whenever $l>L$, which is the case of most upright runners.

\section{Remark 5}

The definition of $\mathcal{S}_{\mathrm{S} \rightarrow \mathrm{f}}$ as in Theorem 1 means that liftoff occurs when the distance between the foot and the COM becomes equal to the nominal leg length of the ES-SLIP, $r_{0}$. $\square$

\section{Remark 6}

To help develop some intuition on Theorem 1, it is noted that the two-dimensional state vector $\eta$ corresponds to the output dynamics; in particular, it corresponds to the pitch error dynamics. The four-dimensional state vector $z$ is suitable for describing the associated zero dynamics. The theorem provides conditions under which, for sufficiently fast exponentially contracting pitch error dynamics, an exponentially stable periodic orbit of the restriction dynamics is also an exponentially stable orbit of the ASLIP. Furthermore, the restriction dynamics, which corresponds to the translational dynamics of the COM of the ASLIP, is rendered diffeomorphic to the ES-SLIP dynamics. Intuitively, the feedback laws of Theorem 1 "coordinate" the actuated degrees of freedom of the ASLIP so that a lower-dimensional subsystem, more specifically the ES-SLIP, "emerges" from the closed-loop dynamics, and it governs the behavior -i.e. the existence and stability properties of periodic orbits of interest- of the fullorder ASLIP.

\section{Remark 7}

The importance of Corollary 1 is that, for given controllers that create an exponentially stable periodic orbit of the ES-SLIP, the feedback laws $u=\Gamma_{\mathrm{c}}^{\epsilon}\left(x_{\mathrm{s}}\right)$ and $\alpha_{\mathrm{f}}=\Gamma_{\mathrm{f}}\left(x_{\mathrm{s}}^{-}\right)$of Theorem 1 render this orbit exponentially stable in the ASLIP.

\section{PROOF OF THE SLIP-EMBEDdING THEOREM}

In this section, Theorem 1 is proved through a sequence of Lemmas. The procedure is constructive, and results in a control law satisfying the requirements of Theorem 1. Fig. 4 summarizes the continuous-time control action during the ASLIP stance phase, whose objective is to render the translational dynamics of the ASLIP COM diffeomorphic to the ES-SLIP dynamics.

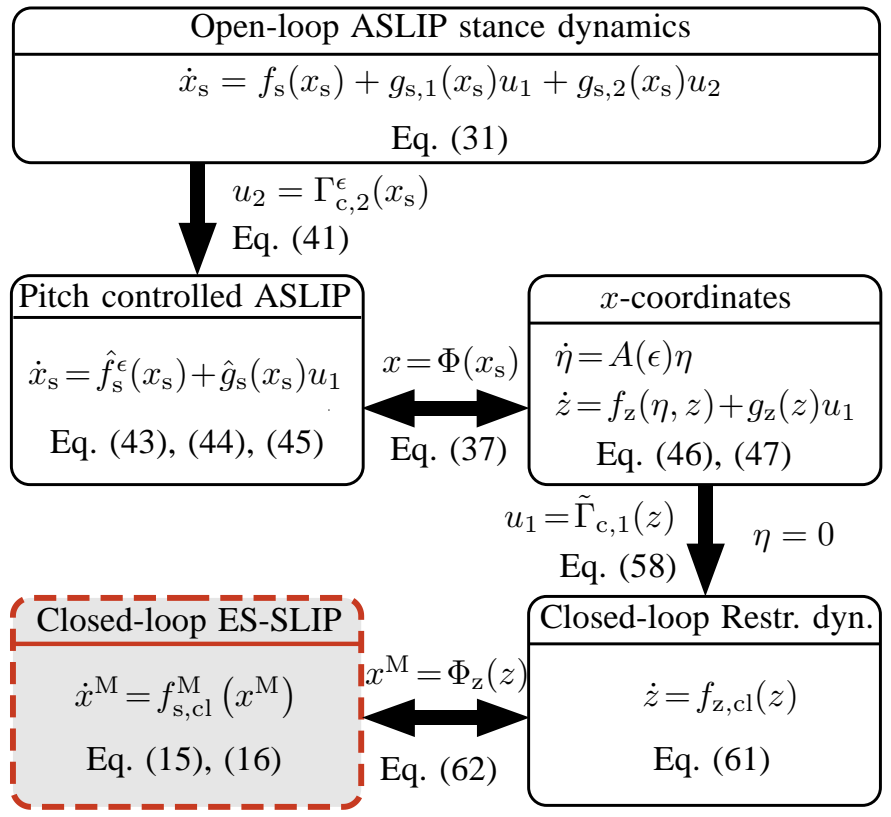

Fig. 4. A diagram summarizing the control procedure through which the ASLIP restriction dynamics is rendered diffeomorphic to the ES-SLIP closedloop dynamics. Vertical arrows correspond to control actions; horizontal arrows relate diffeomorphic dynamics. The dashed box includes the ES-SLIP closed-loop target dynamics. Equation numbers refer to the text.

\section{A. In-stride Continuous Control}

The purpose of the in-stride control action during the stance phase is twofold. First, it ensures that the torso remains at a desired (constant and upright) pitch angle, and second, it renders the translational stance dynamics of the ASLIP diffeomorphic to the ES-SLIP closed-loop stance dynamics. In view of the underactuated nature of the stance phase, the two control objectives will be achieved in different time scales. Since the requirement for the torso being upright throughout the motion is more stringent, high-gain control will be imposed on the pitch rotational motion. Hence, the system will be decomposed into fast and slow dynamics governing the rotational and the translational dynamics of the torso, respectively.

The continuous part of $\Sigma^{\mathrm{ASLIP}}$ in (12), can be written as

$$
\dot{x}_{\mathrm{s}}=f_{\mathrm{s}}\left(x_{\mathrm{s}}\right)+g_{\mathrm{s}, 1}\left(x_{\mathrm{s}}\right) u_{1}+g_{\mathrm{s}, 2}\left(x_{\mathrm{s}}\right) u_{2} .
$$

Define the output $h: \hat{Q}_{\mathrm{s}} \rightarrow \mathbb{R}$ by

$$
y:=h\left(q_{\mathrm{s}}\right):=\theta-\bar{\theta},
$$

where $\bar{\theta}$ is a desired pitch angle, taken to be a constant ${ }^{6}$. The

\footnotetext{
${ }^{6}$ It can rigorously be shown that $\bar{\theta}$ being constant is a necessary condition for the existence of an embedding control law. Due to limited space, the proof of this statement will not be presented here.
} 
output defined by (32) results in the second-order input-output dynamics

$$
\frac{d^{2} y}{d t^{2}}=\left[L_{f_{\mathrm{s}}}^{2} h\left(x_{\mathrm{s}}\right)+L_{g_{\mathrm{s}, 1}} L_{f_{\mathrm{s}}} h\left(q_{\mathrm{s}}\right) u_{1}\right]+L_{g_{\mathrm{s}, 2}} L_{f_{\mathrm{s}}} h\left(q_{\mathrm{s}}\right) u_{2}
$$

where

$$
\begin{gathered}
L_{f_{\mathrm{s}}}^{2} h\left(x_{\mathrm{s}}\right)=0, \\
L_{g_{\mathrm{s}, 1}} L_{f_{\mathrm{s}}} h\left(q_{\mathrm{s}}\right)=\frac{-L \cos \varphi}{J}, L_{g_{\mathrm{s}, 2}} L_{f_{\mathrm{s}}} h\left(q_{\mathrm{s}}\right)=\frac{L \sin \varphi-l}{J l} .
\end{gathered}
$$

Equation (33) shows that two inputs are available for zeroing the (single) output (32). In what follows, the hip torque $u_{2}$ is solely devoted to pitch control, while the leg input $u_{1}$ is reserved for controlling the zero dynamics.

\section{Lemma 1 (Stance Phase Zero Dynamics)}

Under the output function $h$ defined by (32), and for $q_{\mathrm{s}} \in$ $\hat{Q}_{\mathrm{s}}:=\left\{q_{\mathrm{s}} \in Q_{\mathrm{s}} \mid l \neq L \sin \varphi\right\}$,

1) the set $\mathcal{Z}:=\left\{x_{\mathrm{s}}=\left(q_{\mathrm{s}}^{\prime}, \dot{q}_{\mathrm{s}}^{\prime}\right)^{\prime} \in T \hat{Q}_{\mathrm{s}} \mid h\left(q_{\mathrm{s}}\right)=0, L_{f_{\mathrm{s}}} h\left(x_{\mathrm{s}}\right)=\right.$ $0\}$ is a smooth four-dimensional embedded submanifold of $T \hat{Q}_{\mathrm{s}}$;

2) the feedback control law

$$
u_{2}^{*}=-\frac{L_{g_{\mathrm{s}, 1}} L_{f_{\mathrm{s}}} h\left(q_{\mathrm{s}}\right)}{L_{g_{\mathrm{s}, 2}} L_{f_{\mathrm{s}}} h\left(q_{\mathrm{s}}\right)} u_{1}
$$

renders $\mathcal{Z}$ invariant under the stance dynamics; that is, for $x_{\mathrm{s}} \in \mathcal{Z}, u_{1} \in \mathbb{R}$,

$$
f_{\mathrm{s}}\left(x_{\mathrm{s}}\right)+g_{\mathrm{s}, 1}\left(x_{\mathrm{s}}\right) u_{1}+g_{\mathrm{s}, 2}\left(x_{\mathrm{s}}\right) u_{2}^{*} \in T_{x_{\mathrm{s}}} \mathcal{Z}
$$

3 ) there exist smooth functions $\gamma_{1}\left(x_{\mathrm{s}}\right)$ and $\gamma_{2}\left(x_{\mathrm{s}}\right)$ so that the $\operatorname{map} \Phi: T \hat{Q}_{\mathrm{s}} \rightarrow \mathbb{R}^{6}$,

$$
\Phi\left(x_{\mathrm{s}}\right)=:\left(\eta_{1}, \eta_{2}, z_{1}, z_{2}, z_{3}, z_{4}\right)^{\prime}=: x,
$$

where

$$
\begin{gathered}
\eta_{1}:=h\left(q_{\mathrm{s}}\right), \eta_{2}:=L_{f_{\mathrm{s}}} h\left(x_{\mathrm{s}}\right), \\
\left(z_{1}, z_{2}\right)^{\prime}:=(l, \varphi)^{\prime},\left(z_{3}, z_{4}\right)^{\prime}:=\left(\gamma_{1}\left(x_{\mathrm{s}}\right), \gamma_{2}\left(x_{\mathrm{s}}\right)\right)^{\prime},
\end{gathered}
$$

is a valid coordinate transformation, i.e. $\Phi$ is a diffeomorphism onto its image, and

$$
L_{g_{\mathrm{s}, 2}} \gamma_{1}\left(x_{\mathrm{s}}\right)=0, L_{g_{\mathrm{s}, 2}} \gamma_{2}\left(x_{\mathrm{s}}\right)=0
$$

4) the set $\mathcal{S}_{\mathrm{s} \rightarrow \mathrm{f}} \cap \mathcal{Z}$ with $\mathcal{S}_{\mathrm{s} \rightarrow \mathrm{f}}$ defined by (8) is a co-dimension one $C^{1}$-submanifold of $\mathcal{Z}$.

\section{Proof}

Parts 1) and 2) of Lemma 1 follow from general results in [25, pp. 169-170]. For part 3), consider the distribution $G:=\operatorname{span}\left\{g_{\mathrm{s}, 2}\right\}$, which has constant dimension $d=1$ on $T \hat{Q}_{\mathrm{s}}$. Since $G$ is one dimensional, it is involutive, and thus, by the Frobenius theorem (Theorem 1.4.1, [25, p. 23]), integrable. As a result there exist $n-d=6-1=5$ realvalued functions defined on $T \hat{Q}_{\mathrm{s}}$ such that the annihilator of $G$ is $G^{\perp}=\operatorname{span}\left\{d l, d \varphi, d \theta, d \gamma_{1}, d \gamma_{2}\right\}$. A straightforward application of the constructive proof of the sufficiency part of Frobenius theorem [25, pp. 24-28] results in

$$
\begin{gathered}
\gamma_{1}\left(x_{\mathrm{s}}\right)=\dot{l}+(L \cos \varphi) \dot{\theta}, \\
\gamma_{2}\left(x_{\mathrm{s}}\right)=\dot{\varphi}-\left[-1+\frac{L \sin \varphi}{l}+\frac{J}{m l(L \sin \varphi-l)}\right] \dot{\theta} .
\end{gathered}
$$

It is straightforward to check that $\Phi$ is a diffeomorphism onto its image in $\mathbb{R}^{6}$. Finally, for part 4), note that, in $x$-coordinates, $\tilde{H}_{\mathrm{s} \rightarrow \mathrm{f}}(x):=\left(H_{\mathrm{s} \rightarrow \mathrm{f}} \circ \Phi^{-1}\right)(x)=r_{0}-$ $\sqrt{L^{2}+z_{1}^{2}-2 L z_{1} \sin z_{2}}$, i.e. $\tilde{H}_{\mathrm{s} \rightarrow \mathrm{f}}$ is a function of $z$ only. In particular, it does not depend on $\eta$ and $\dot{\eta}$. The result now follows from the regular value theorem (Theorem (5.8) of [8, p. 78]), in view of Remark 1 and of the fact that $\operatorname{rank}\left\{\left(h, L_{f_{\mathrm{s}}} h, H_{\mathrm{s} \rightarrow \mathrm{f}}\right)^{\prime}\right\}=2+\operatorname{rank}\left\{H_{\mathrm{s} \rightarrow \mathrm{f}}\right\}=3$.

It should be noted that, contrary to the HZD designed in [48] and [12], the zero dynamics manifold $\mathcal{Z}$ is a four-dimensional embedded submanifold of the six-dimensional stance state space $T \hat{Q}_{\mathrm{s}}$. This significantly complicates stability analysis of the resulting HZD, which no longer is a one-DOF system as in [48] and [12]. However, the presence of $u_{1}$ in the zero dynamics allows for further control action. A feedback law can be devised for $u_{1}$ so that the zero dynamics associated with the output (32) matches exactly the differential equations of the ES-SLIP stance phase dynamics. To do this, let $\epsilon>0$ and define the feedback

$$
\begin{aligned}
u_{2} & =\Gamma_{\mathrm{c}, 2}^{\epsilon}\left(x_{\mathrm{s}}\right) \\
& :=\frac{1}{L_{g_{\mathrm{s}, 2}} L_{f_{\mathrm{s}}} h\left(q_{\mathrm{s}}\right)}\left[v^{\epsilon}(\theta, \dot{\theta})-L_{g_{\mathrm{s}, 1}} L_{f_{\mathrm{s}}} h\left(q_{\mathrm{s}}\right) u_{1}\right],
\end{aligned}
$$

where

$$
v^{\epsilon}(\theta, \dot{\theta}):=-\frac{1}{\epsilon^{2}} K_{P}^{\theta}(\theta-\bar{\theta})-\frac{1}{\epsilon} K_{V}^{\theta} \dot{\theta},
$$

and $K_{P}^{\theta}, K_{V}^{\theta}$ are positive constants. Under this feedback law, the model (31) becomes

$$
\dot{x}_{\mathrm{s}}=\hat{f}_{\mathrm{s}}^{\epsilon}\left(x_{\mathrm{s}}\right)+\hat{g}_{\mathrm{s}}\left(x_{\mathrm{s}}\right) u_{1},
$$

where

$$
\begin{gathered}
\hat{f}_{\mathrm{s}}^{\epsilon}\left(x_{\mathrm{s}}\right):=f_{\mathrm{s}}\left(x_{\mathrm{s}}\right)+\left[\frac{1}{L_{g_{\mathrm{s}, 2}} L_{f_{\mathrm{s}}} h\left(q_{\mathrm{s}}\right)} v^{\epsilon}(\theta, \dot{\theta})\right] g_{\mathrm{s}, 2}\left(x_{\mathrm{s}}\right), \\
\hat{g}_{\mathrm{s}}\left(x_{\mathrm{s}}\right):=g_{\mathrm{s}, 1}\left(x_{\mathrm{s}}\right)-\frac{L_{g_{\mathrm{s}, 1}} L_{f_{\mathrm{s}}} h\left(q_{\mathrm{s}}\right)}{L_{g_{\mathrm{s}, 2}} L_{f_{\mathrm{s}}} h\left(q_{\mathrm{s}}\right)} g_{\mathrm{s}, 2}\left(x_{\mathrm{s}}\right) .
\end{gathered}
$$

In the coordinates of Lemma 1, (43) has the form

$$
\begin{gathered}
\dot{\eta}=A(\epsilon) \eta, \\
\dot{z}=f_{\mathrm{z}}(\eta, z)+g_{\mathrm{z}}(z) u_{1},
\end{gathered}
$$

where

$$
A(\epsilon)=\left(\begin{array}{cc}
0 & 1 \\
-K_{P}^{\theta} / \epsilon^{2} & -K_{V}^{\theta} / \epsilon
\end{array}\right) .
$$

With the additional change of coordinates $\eta=\Pi(\epsilon) \tilde{\eta}$, defined by $\eta_{1}=\epsilon \tilde{\eta}_{1}$ and $\eta_{2}=\tilde{\eta}_{2}$, the model (46)-(47) takes the form

$$
\begin{gathered}
\epsilon \dot{\tilde{\eta}}=\tilde{A} \tilde{\eta}, \\
\dot{z}=f_{\mathrm{z}}(\Pi(\epsilon) \tilde{\eta}, z)+g_{\mathrm{z}}(z) u_{1},
\end{gathered}
$$

and

$$
\frac{1}{\epsilon} \tilde{A}=\Pi^{-1}(\epsilon) A(\epsilon) \Pi(\epsilon) \Rightarrow \tilde{A}=\left(\begin{array}{cc}
0 & 1 \\
-K_{P}^{\theta} & -K_{V}^{\theta}
\end{array}\right) .
$$

Since the gains $\left\{K_{P}^{\theta}, K_{V}^{\theta}\right\}$ in (51) are strictly positive, the matrix $\tilde{A}$ is Hurwitz and $e^{\frac{1}{\epsilon} \tilde{A}}$ converges to zero exponentially fast as $\epsilon \rightarrow 0$. Hence, $\lim _{\epsilon \backslash 0} e^{A(\epsilon)}=0$. This verifies condition 
A.3) of Theorem 1. Moreover, setting $\epsilon=0$, (49) reduces to the algebraic equation $\tilde{A} \tilde{\eta}=0$, which has the origin as its unique solution. Hence, (49)-(50) is in standard singular perturbation form, see [26, p. 424], and the corresponding reduced model is obtained by substituting $\epsilon=0$ and $\tilde{\eta}=0$ in the slow part of the dynamics (50), i.e.

$$
\dot{z}=f_{\mathrm{z}}(0, z)+g_{\mathrm{z}}(z) u_{1},
$$

where direct calculation leads to

$$
\begin{gathered}
f_{\mathrm{z}}(z)=\left(\begin{array}{c}
z_{3} \\
z_{4} \\
z_{1} z_{4}^{2}-g \cos \left(\bar{\theta}+z_{2}\right) \\
\frac{-2 z_{3} z_{4}+g \sin \left(\bar{\theta}+z_{2}\right)}{z_{1}}
\end{array}\right), \\
g_{\mathrm{z}}(z)=\left(\begin{array}{c}
0 \\
0 \\
1 / m \\
\frac{L \cos z_{2}}{m z_{1}\left(L \cos z_{2}-z_{1}\right)}
\end{array}\right) .
\end{gathered}
$$

The following lemma completes the continuous stance controller design by providing a procedure for constructing $u_{1}$.

\section{Lemma 2 (Restriction dynamics)}

If $\bar{\theta}$ is the desired pitch angle in (32), define

$$
\begin{gathered}
r(z):=\sqrt{L^{2}+z_{1}^{2}-2 L z_{1} \sin z_{2}}, \\
\dot{r}(z):=\frac{z_{1}-L \sin z_{2}}{r(z)} z_{3}-\frac{L z_{1} \cos z_{2}}{r(z)} z_{4}, \\
y_{z}(z):=z_{1} \cos \left(z_{2}+\bar{\theta}\right)+L \sin \bar{\theta} .
\end{gathered}
$$

Then, if $\bar{E}$ is the desired energy level, the feedback law

$$
u_{1}=\tilde{\Gamma}_{\mathrm{c}, 1}(z):=\frac{z_{1}-L \sin z_{2}}{r(z)} F_{\mathrm{ES}-\mathrm{SLIP}}(z),
$$

with

$$
\begin{aligned}
& F_{\mathrm{ES}-\mathrm{SLIP}}(z):=k\left[r_{0}+\Delta r-r(z)\right]-K_{P}^{E} \dot{r}(z)[E(z)-\bar{E}], \\
& E(z):=\frac{1}{2} m\left(z_{3}^{2}+z_{1}^{2} z_{4}^{2}\right)+m g y_{z}(z)+\frac{1}{2} k\left[r_{0}+\Delta r-r(z)\right]^{2},
\end{aligned}
$$

and $K_{P}^{E}>0$, renders the restriction dynamics (52) diffeomorphic to the ES-SLIP closed-loop dynamics $f_{\mathrm{s}, \mathrm{cl}}^{\mathrm{M}}\left(x^{\mathrm{M}}\right)$ defined by (16).

\section{Proof}

Substitution of (58) into (52) gives

$$
\dot{z}=f_{\mathrm{z}}(z)+g_{\mathrm{z}}(z) \tilde{\Gamma}_{\mathrm{c}, 1}(z)=: f_{\mathrm{z}, \mathrm{cl}}(z) .
$$

Define the map $\Phi_{\mathrm{z}}: \mathcal{Z} \rightarrow \mathbb{R}^{4}$ by

$$
\Phi_{\mathrm{z}}(z):=\left(\begin{array}{c}
-z_{1} \sin \left(z_{2}+\bar{\theta}\right)+L \cos \bar{\theta} \\
z_{1} \cos \left(z_{2}+\bar{\theta}\right)+L \sin \bar{\theta} \\
-z_{3} \sin \left(z_{2}+\bar{\theta}\right)-z_{1} z_{4} \cos \left(z_{2}+\bar{\theta}\right) \\
z_{3} \cos \left(z_{2}+\bar{\theta}\right)-z_{1} z_{4} \sin \left(z_{2}+\bar{\theta}\right)
\end{array}\right) .
$$

It is straightforward to check that $\Phi_{\mathrm{z}}$ is a diffeomorphism onto its image, thus it describes a valid coordinate transformation on $\mathcal{Z}$. Observe that $\Phi_{\mathrm{z}}(z)=x^{\mathrm{M}}$. The result

$$
\left.\left(\frac{\partial \Phi_{\mathrm{z}}}{\partial z} f_{\mathrm{z}, \mathrm{cl}}(z)\right)\right|_{z=\Phi_{\mathrm{z}}^{-1}\left(x^{\mathrm{M}}\right)}=f_{\mathrm{s}, \mathrm{cl}}^{\mathrm{M}}\left(x^{\mathrm{M}}\right)
$$

is obtained after straightforward algebraic manipulations.

\section{Remark 8}

Careful inspection of (58) reveals that under the proposed feedback law the total ASLIP leg force, $u_{1}$, becomes equal to the projection of the ES-SLIP force, $F_{\mathrm{ES}-\mathrm{SLIP}}$, along the direction of the actual (ASLIP) leg. As will be explained in Section IX-D, this property can be used to provide a qualitative explanation of the superiority of the SLIP-embedding controller against controllers that create non-compliant HZD.

\section{Remark 9}

Combining (41) and (58), a feedback controller of the form $u=\Gamma_{\mathrm{c}}^{\epsilon}\left(x_{\mathrm{s}}, \alpha_{\mathrm{s}}\right)$ is obtained. The vector $\alpha_{\mathrm{s}}=\left(\bar{\theta}, k, r_{0}, \Delta r\right)^{\prime}$ corresponds to parameters introduced by the control law, and includes the mechanical properties of the target model. The nominal values of these parameters will be selected via an optimization procedure, which will be presented in Section IX. As was mentioned in Section IV, $\alpha_{\mathrm{s}}$ can be updated in an event-based manner through the inner-loop feedback law $\Gamma_{\mathrm{s}}$ of Fig. 2 to achieve hybrid invariance. However, Lemma 3 below shows that this is not necessary for the SLIP-embedding controller, and thus $\alpha_{\mathrm{s}}$ need not be updated. This is the reason why $\alpha_{\mathrm{s}}$ did not explicitly appear as one of the arguments of the continuous-time controller $\Gamma_{\mathrm{c}}^{\epsilon}$.

\section{B. Event-Based Discrete Control}

The purpose of the stride-to-stride controller is twofold. First, it ensures that the manifold $\mathcal{S}_{\mathrm{s} \rightarrow \mathrm{f}} \cap \mathcal{Z}$ is invariant under the reset map $\Delta_{\mathrm{cl}}$. Second, it arranges the configuration of the ASLIP at liftoff so that the restriction of the ASLIP reset map on $\mathcal{S}_{\mathrm{s} \rightarrow \mathrm{f}} \cap \mathcal{Z}$ is equal to the SLIP closed-loop reset map. Both requirements can be satisfied through the outer-loop eventbased controller $\Gamma_{\mathrm{f}}$ of Fig. 2, the design of which is the subject of the following lemma.

\section{Lemma 3 (Event-based controller)}

Let $\dot{\bar{x}}_{\mathrm{c}}$ and $\bar{\psi}$ be the forward running speed at liftoff and the touchdown angle, respectively, corresponding to a (desired) fixed point of the ES-SLIP. Define

$$
\psi\left(x_{\mathrm{s}}^{-}\right):=\bar{\psi}+K_{\dot{x}}\left[\dot{x}_{\mathrm{c}}^{-}\left(x_{\mathrm{s}}^{-}\right)-\dot{\bar{x}}_{\mathrm{c}}\right],
$$

where $\dot{x}_{\mathrm{c}}^{-}$is the forward running speed of the ASLIP prior to liftoff. Then, the controller $\alpha_{\mathrm{f}}=\Gamma_{\mathrm{f}}\left(x_{\mathrm{s}}^{-}\right)=$ $\left(l^{\mathrm{td}}\left(x_{\mathrm{s}}^{-}\right), \varphi^{\mathrm{td}}\left(x_{\mathrm{s}}^{-}\right)\right)^{\prime}$,

$$
\begin{array}{r}
l^{\mathrm{td}}\left(x_{\mathrm{s}}^{-}\right)=\sqrt{L^{2}+r_{0}^{2}+2 L r_{0} \sin \left(\psi\left(x_{\mathrm{s}}^{-}\right)-\bar{\theta}\right)}, \\
\varphi^{\mathrm{td}}\left(x_{\mathrm{s}}^{-}\right)=\arcsin \left[\frac{\left(l^{\mathrm{td}}\left(x_{\mathrm{s}}^{-}\right)\right)^{2}+L^{2}-r_{0}^{2}}{2 L l^{\mathrm{td}}\left(x_{\mathrm{s}}^{-}\right)}\right],
\end{array}
$$

where $\bar{\theta}$ is the desired pitch angle in (32), achieves B.1) and B.2) of Theorem 1. 


\section{Proof}

Suppose $x_{\mathrm{s}}^{-} \in \mathcal{S}_{\mathrm{s} \rightarrow \mathrm{f}} \cap \mathcal{Z}$. To show B.1), notice that this implies $\dot{\theta}^{-}=0$ and $\theta^{-}=\bar{\theta}$ just prior to liftoff. Since during the flight phase $\ddot{\theta}=0$, i.e. $\theta(t) \equiv \bar{\theta}$, at touchdown we have $\dot{\theta}^{+}=0$ and $\theta^{+}=\bar{\theta}$, which means that $x_{\mathrm{s}}^{+} \in \mathcal{Z}$. This establishes hybrid invariance, i.e. $\Delta_{\mathrm{cl}}\left(\mathcal{S}_{\mathrm{s} \rightarrow \mathrm{f}} \cap \mathcal{Z}\right) \subset \mathcal{Z}$. To show B.2), observe that, in coordinates (62), the surface $\mathcal{S}_{\mathrm{s} \rightarrow \mathrm{f}} \cap \mathcal{Z}$ with $\mathcal{S}_{\mathrm{s} \rightarrow \mathrm{f}}$ defined by (8), is equal to $\mathcal{S}_{\mathrm{s} \rightarrow \mathrm{f}}^{\mathrm{M}}$, given by (19), i.e. the domains of definition of the maps $\Delta_{\mathrm{cl}} \mathcal{Z}$ and $\Delta_{\mathrm{cl}}^{\mathrm{M}}$ are equal. The rest of the proof is a consequence of the fact that the flight flow of the ES-SLIP is the same as the translational part of the flight flow of the ASLIP. Equations (64)-(66) ensure that, not only the flight flows are identical, but also the corresponding closed-loop reset maps $\left.\Delta_{\mathrm{cl}}\right|_{\mathcal{Z}}$ and $\Delta_{\mathrm{cl}}^{\mathrm{M}}$, are diffeomorphic.

\section{Remark 10}

The proof of Lemma 3 depends only upon the restriction of the functions $l^{\text {td }}$ and $\varphi^{\text {td }}$ on $\mathcal{S}_{\mathrm{s} \rightarrow \mathrm{f}} \cap \mathcal{Z}$. Hence, $l^{\text {td }}$ and $\varphi^{\text {td }}$ can be replaced with any smooth functions whose restrictions on $\mathcal{S}_{\mathrm{s} \rightarrow \mathrm{f}} \cap \mathcal{Z}$ are equal to (65) and (66), respectively. This property will be brought into use in Section IX-A to modify (65) and (66) in order to enlarge the basin of attraction of the nominal orbit; see (72) and (73) there.

\section{Proof of Theorem 1}

The proof of Theorem 1 is an immediate consequence of Lemmas 1, 2 and 3.

\section{One DOF Hybrid Zero DynAmics: The Rigid TARGET MODEL}

This section describes the second of the controllers presented in this paper. The design procedure provides the feedback laws $\Gamma_{\mathrm{c}}, \Gamma_{\mathrm{s}}$ and $\Gamma_{\mathrm{f}}$, whose function was described in Section IV. This controller, whose stability proof follows from previous results in [12] and [32], is included here because its comparison with the SLIP-embedding controller will reveal some beneficial aspects of designing the HZD to accommodate compliance. Thus, the presentation will be terse; the interested reader is referred to [35] for particular details on the control design, and in [32] for the general framework. It is important to emphasize that this controller is fundamentally different from the SLIP-embedding controller of Sections VI and VII in that it results in a one-DOF HZD, a fact that greatly simplifies stability analysis, but leaves no room for compliance. Hence, we refer to this controller as the rigid target model controller.

\section{A. In-stride Continuous Control}

During the stance phase, the ASLIP exhibits one degree of underactuation. The two inputs $u=\left(u_{1}, u_{2}\right)^{\prime}$ will be used to asymptotically impose two virtual holonomic constraints on two of the models' three DOF, which are chosen to be the leg length and the pitch angle, i.e. $q_{\mathrm{a}}=(l, \theta)^{\prime}$. Other choices are possible; however, this particular one allows for the direct comparison with the SLIP-embedding controller of Sections VI and VII. Here, the virtual constraints are chosen to be polynomials parameterized by the monotonic quantity $q_{\mathrm{u}}=\pi / 2-\varphi-\theta$, representing the angle of the leg with respect to the ground, as shown in Fig. 1. The virtual constraints are imposed through zeroing the output

$$
y=h\left(q_{\mathrm{s}}, \alpha_{\mathrm{s}}\right)=q_{\mathrm{a}}-h_{\mathrm{d}}\left(q_{\mathrm{u}}, \alpha_{\mathrm{s}}\right),
$$

where $h_{\mathrm{d}}$ are the polynomial functions of $q_{\mathrm{u}}$ describing the desired evolution of $q_{\mathrm{a}}$, and $\alpha_{\mathrm{s}}$ includes the corresponding polynomial coefficients; see [35, Appendix].

Following the procedure that was outlined in Section IV, and is further detailed in [35, Section III-B], the continuous feedback controller $\Gamma_{\mathrm{c}}$ is designed to render the surface

$$
\mathcal{Z}_{\alpha_{\mathrm{s}}}=\left\{x_{\mathrm{s}} \in T Q_{\mathrm{s}} \mid h\left(q_{\mathrm{s}}, \alpha_{\mathrm{s}}\right)=0, L_{f_{\mathrm{s}}} h\left(x_{\mathrm{s}}, \alpha_{\mathrm{s}}\right)=0\right\}
$$

invariant under the flow of the continuous part of the ASLIP dynamics and attractive. It is emphasized here that two virtual constraints are imposed by zeroing (67), thus resulting in a one-DOF HZD evolving on a two-dimensional surface $\mathcal{Z}_{\alpha_{\mathrm{s}}}$.

\section{B. Event-Based Discrete Control}

The development of the event-based control law closely follows the structure outlined in Section IV. In this case, to achieve hybrid invariance, it is necessary to include the innerloop controller $\Gamma_{\mathrm{s}}$ of Fig. 2 in the feedback design. Details on how to construct $\Gamma_{\mathrm{s}}$ can be found in [35, Section III-C].

The outer-loop control law $\Gamma_{\mathrm{f}}$ updates $\alpha_{\mathrm{f}}=\left(l^{\mathrm{td}}, \varphi^{\mathrm{td}}\right)^{\prime}$ in order to exponentially stabilize the HZD. In the rigid target model controller, we do not explore the possibility of updating the leg length $l^{\text {td }}$ at touchdown; $l^{\text {td }}$ is assumed to be always equal to its nominal value $l_{0}$. This leaves the touchdown angle $\varphi^{\mathrm{td}}$ as the only parameter available for control. The Poincaré map $\mathcal{P}$ associated with the hybrid system under the feedback laws $\Gamma_{\mathrm{c}}$ and $\Gamma_{\mathrm{s}}$ gives rise to the discrete-time control system,

$$
x_{\mathrm{s}}^{-}(k+1)=\mathcal{P}\left(x_{\mathrm{s}}^{-}(k), \varphi^{\mathrm{td}}(k)\right),
$$

defined on the surface

$$
\mathcal{S}_{\mathrm{s} \rightarrow \mathrm{f}}^{\prime}=\left\{x_{\mathrm{s}} \in \mathcal{X}_{\mathrm{s}} \mid l-l_{0}=0, i>0\right\},
$$

where $x_{\mathrm{s}}^{-}(k)$ is the state just prior to the k-th liftoff. Linearizing (69) and implementing a discrete LQR controller gives

$$
\varphi^{\mathrm{td}}(k)=\Gamma_{\mathrm{f}}\left(x_{\mathrm{s}}^{-}(k)\right)=\bar{\varphi}^{\mathrm{td}}+K\left[x_{\mathrm{s}}^{-}(k)-\bar{x}_{\mathrm{s}}^{-}\right],
$$

where $\bar{x}_{\mathrm{s}}^{-}$and $\bar{\varphi}^{\mathrm{td}}$ are the nominal values of the state just prior to the k-th liftoff and of the touchdown angle, respectively. The feedback controller (71) guarantees that the eigenvalues of the linearization of (69) are all within the unit circle, and completes the control design. Note that instead of the full model Poincaré map (69), the one-dimensional Poincaré map associated with the HZD could have been used, affording a reduced-order stability test; see [48], [12], [32].

\section{Controller Evaluation via Simulation}

This section presents simulation results that compare the performance of the SLIP-embedding controller presented in Sections VI and VII, with that of the rigid target model controller of Section VIII. Both the steady-state and the transient behaviors of the two controllers are discussed. 


\section{A. Implementation Issues and Nominal Orbit Design}

The mechanical properties of the ASLIP used in the simulations roughly correspond to robotic testbeds constructed in a collaborative effort between the University of Michigan and Carnegie Mellon University, and are presented in Table I (see also Fig. 1).

TABLE I

ASLIP MECHANICAL PARAMETERS

\begin{tabular}{lcr}
\hline Parameter & Value & Units \\
\hline Torso Mass $(m)$ & 27 & $\mathrm{~kg}$ \\
Torso Inertia $(J)$ & 1 & $\mathrm{~kg} \mathrm{~m}^{2}$ \\
Hip-to-COM spacing $(L)$ & 0.25 & $\mathrm{~m}$ \\
Nominal Leg Length $\left(l_{0}\right)$ & 0.9 & $\mathrm{~m}$ \\
Uncompressed Spring Length $\left(l_{\text {nat }}\right)$ & 0.91 & $\mathrm{~m}$ \\
ASLIP Spring Constant $\left(k_{\mathrm{A}}\right)$ & 7578 & $\mathrm{~N} / \mathrm{m}$ \\
\hline
\end{tabular}

In implementing the SLIP-embedding controller, simulation shows that, while the event-based controller developed in Lemma 3 of Section VII-B achieves exponential stability of the ASLIP, letting the pitch angle in (65)-(66) off the zero dynamics be equal to its actual value, instead of its nominal value $\bar{\theta}$, enlarges the domain of attraction of the controller, i.e.

$$
\begin{aligned}
& l^{\mathrm{td}}\left(x_{\mathrm{f}}, x_{\mathrm{s}}^{-}\right)=\sqrt{L^{2}+r_{0}^{2}+2 L r_{0} \sin \left(\psi\left(x_{\mathrm{s}}^{-}\right)-\theta\right)}, \\
& \varphi^{\mathrm{td}}\left(x_{\mathrm{f}}, x_{\mathrm{s}}^{-}\right)=\arcsin \left[\frac{\left(l^{\mathrm{td}}\left(x_{\mathrm{f}}, x_{\mathrm{s}}^{-}\right)\right)^{2}+L^{2}-r_{0}^{2}}{2 L l^{\mathrm{td}}\left(x_{\mathrm{f}}, x_{\mathrm{s}}^{-}\right)}\right],
\end{aligned}
$$

whose restrictions on $\mathcal{S}_{\mathrm{s} \rightarrow \mathrm{f}} \cap \mathcal{Z}$ are equal to (65) and (66), respectively. By Remark 10, the stability conclusion of Theorem 1 remains valid. This modification is similar to what was done in [12], and it will be included in the simulations of the SLIP-embedding controller without further comment.

To implement the rigid target model controller, a sixth order polynomial was used for the desired leg length, and a constant polynomial for the desired pitch angle; refer to [35, Appendix] for details. Generally, the rigid target model controller allows for the desired pitch angle $\theta$ being any suitably parameterized function of the unactuated variable $q_{\mathrm{u}}$, thus allowing for nontrivial motions of the torso. However, this is not possible in the SLIP-embedding controller, due to the fact that constant pitch angle throughout the nominal (steady-state) motion is a necessary condition for its implementation.

Both controllers introduce a set of parameters $\alpha_{\mathrm{s}}$, whose values along the nominal orbit can be selected using the optimization technique developed in [48]. Consider the hybrid dynamics of the ASLIP in closed-loop with the feedback controllers developed in Sections VI and VII, and in Section VIII with cost function

$$
\begin{aligned}
\hat{J}\left(\alpha_{\mathrm{s}}\right) & =\frac{1}{T_{\mathrm{s}}} \int_{0}^{T_{\mathrm{s}}} u_{2}^{2}(t) d t \\
& +\max _{t \in\left[0, T_{\mathrm{s}}\right]}\left\{\left[u_{1}(t)-k_{\mathrm{A}}\left(l_{\text {nat }}-l(t)\right)\right]^{2}\right\},
\end{aligned}
$$

where $T_{\mathrm{s}}$ is the duration of the stance phase, $k_{\mathrm{A}}$ is the stiffness of the ASLIP leg, and $l_{\text {nat }}$ its natural length; see Table I.
Append to (74) the constraint

$$
x_{\mathrm{s}}^{-}-\mathcal{P}\left(x_{\mathrm{s}}^{-}, \alpha_{\mathrm{s}}, \alpha_{\mathrm{f}}\right)=0,
$$

so that the nominal orbit is periodic. One can also include constraints that correspond to requirements such as the desired nominal forward speed, or the normal ground force component be non-negative, etc. Then, the problem of finding the nominal values of the coefficients $\alpha_{\mathrm{s}}$ and $\alpha_{\mathrm{f}}$ reduces to a constrained minimization problem, which can be (numerically) solved using MATLAB's fmincon. It worth mentioning here, that the specific choice of performance index (74) reflects our desire to find a nominal orbit for the ASLIP, on which the amount of work produced by the hip actuator and the peak force developed by the leg actuator given by

$$
u_{1}^{\mathrm{a}}=u_{1}-k_{\mathrm{A}}\left(l_{\mathrm{nat}}-l\right) \text {, }
$$

are minimized.

\section{B. Steady-State Behavior}

In order to compare the behavior of the two controllers under perturbations, it would be ideal to have identical nominal orbits. Despite the fact that relatively low degree polynomials have been used in the rigid target model controller, an almost exact match in the resulting nominal orbits was obtained, as depicted in Fig. 5. Fig. 5 also shows that both controllers take advantage of the leg spring on the nominal (steady-state) motion, since the leg actuator force $u_{1}^{\mathrm{a}}$ is below $6 N$ while the total leg forces are on the order of $900 \mathrm{~N}$ in both cases.

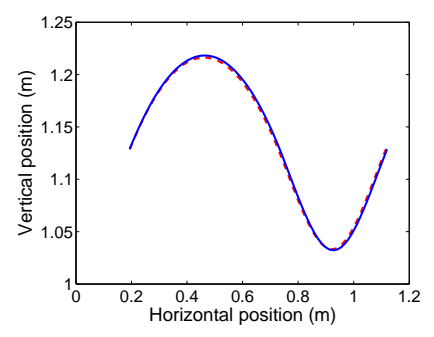

(a)

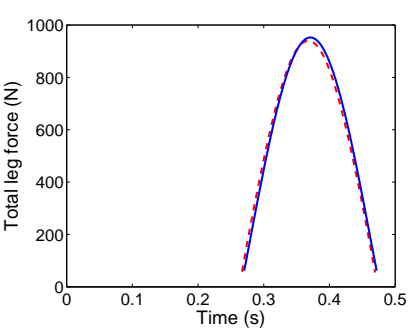

(c)

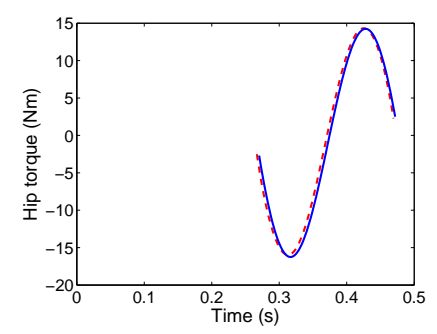

(b)

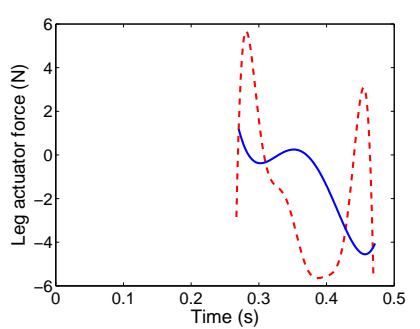

(d)
Fig. 5. Nominal orbits in physical space (a), and corresponding hip torques (b), total leg forces (c), and leg actuator forces (d) computed by (76), for the rigid target model controller (dashed lines) and the SLIP-embedding controller (solid lines).

\section{Transient Behavior and Performance Evaluation}

The gains used in the SLIP-embedding controller are $K_{P}^{\theta}=300, K_{V}^{\theta}=2 \sqrt{K_{P}^{\theta}}, \epsilon=1.2, K_{P}^{E}=2$, and $K_{\dot{x}}=0.2$, 
while the gains for the rigid target model controller are

$$
\begin{gathered}
K_{P}^{y}=\operatorname{diag}\{100,100\}, K_{V}^{y}=2 \sqrt{K_{P}^{y}}, \epsilon=1, \text { and } \\
K=(0.1839,0.4555,-0.0048,0.0887,0.1902) .
\end{gathered}
$$

Note that $K$ has been selected using MATLAB's dlqr on the discrete system (69) evolving on the Poincare section (70). The specific values were chosen such that these two controllers exhibit similar behavior in response to a perturbation in the pitch angle; see Fig. 6-(a) and (b).

Using these data, both controllers have been simulated in MATLAB. It was observed that the rigid target model controller tends to violate the unilateral constraint between the ground and the toe by developing control forces which "pull" against the ground (i.e. the normal force becomes negative). To enlarge the domain of attraction, it was necessary to include saturation on the control forces so that the ground constraints are respected; more information on the saturation procedure can be found in supplemental material available in [18]. The SLIP-embedding controller did not violate these constraints, except at very large perturbations.

Fig. 6 presents pitch angle and forward velocity as the ASLIP recovers from a perturbation $\delta \theta=-6 \mathrm{deg}$ using both controllers. The perturbation occurs at the liftoff of the second stride. Notice that in both cases, the response of the pitch angle is similar; however, larger excursions from the nominal forward speed are observed in the rigid target model controller.

Fig. 7 presents the total leg forces and the leg actuator forces corresponding to Fig. 6. It is seen that, in the SLIP-embedding controller, the profile of the total leg forces $u_{1}$ remains close to that of a spring force, even during transients, resulting in small actuator forces $u_{1}^{\mathrm{a}}$ computed by (76). On the contrary, in the rigid target model controller, the profile of the total leg force $u_{1}$ significantly differs from that of the spring force, resulting in large actuator forces $u_{1}^{a}$. This means that the rigid target model controller in closed loop with the ASLIP effectively "cancels" the compliance of the leg in the open-loop ASLIP. It is emphasized that, on the nominal orbit, both controllers exploit the leg spring equally well, since as shown in Fig. 5, the leg actuator force never exceeds $6 N$, while the total forces are on the order of $900 \mathrm{~N}$.

These features have significant implications for the domain of attraction of the two controllers. This is demonstrated in Table II, which presents the number of strides until convergence within $5 \%$ of the steady-state value (strides), the peak actuator forces $\left(u_{1}^{a}, u_{2}\right)^{\max }$ in $N$, and the total work ${ }^{7}\left(W_{1}, W_{2}\right)^{\text {total }}$ in $J$, required to reject perturbations $\delta \theta$ in the pitch angle and $\delta \dot{x}_{\mathrm{c}}$ in the forward velocity using the SLIP-embedding controller (SLIP) and the Rigid Target Model controller (RTM). The perturbations reported in Table II correspond to the maximum values that can be rejected with the RTM controller, while the leg actuator force satisfies $u_{1}^{a} \leq 500 N$ (almost double the weight of the robot). A more complete table that includes perturbations to state variables not presented in Table II can be found in supplemental material available in [18]. As is shown in Table II, significantly lower peak leg actuator forces and total work are required from the SLIP-embedding controller. As a result, larger perturbations than those in Table II can be rejected by the SLIP-embedding controller respecting the constraint $u_{1}^{a} \leq 500 N$. The following section provides a qualitative explanation of this behavior.

\footnotetext{
${ }^{7}$ The total work is computed as the integral of the absolute value of the power injected by the actuators.
}

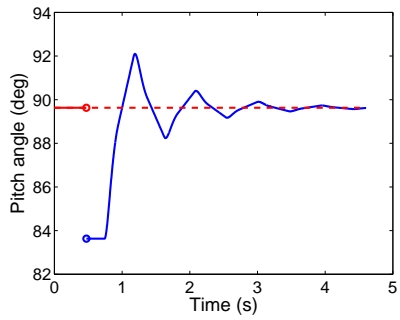

(a)

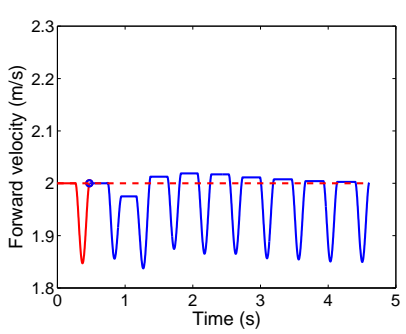

(c)

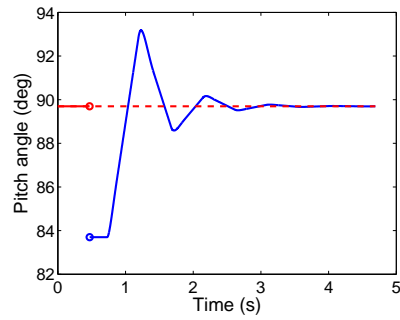

(b)

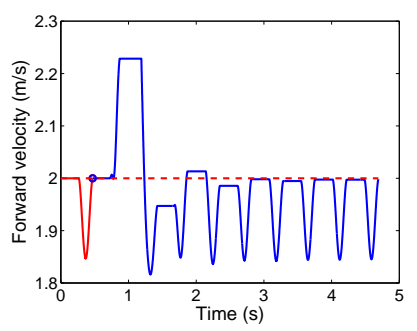

(d)

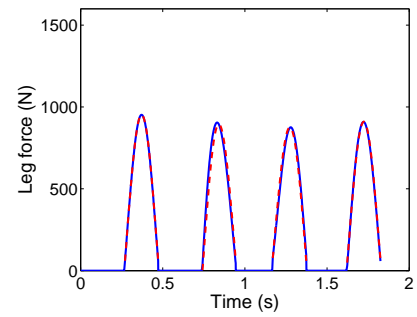

(a)

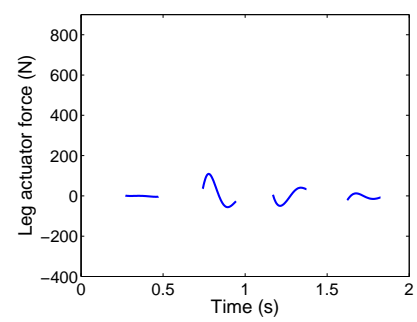

(c)

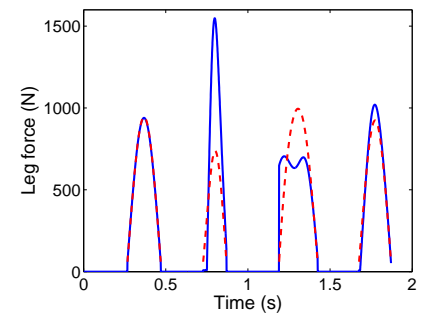

(b)

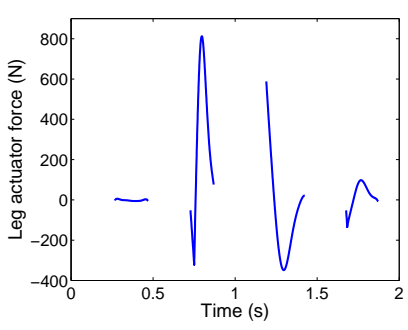

(d)

Fig. 6. Ten strides showing convergence from $\delta \theta=-6 \mathrm{deg}$, for the the SLIPembedding controller (a), (c), and the rigid target model controller (b),(d). Dashed lines show desired values; the circles correspond to the instant when the perturbation occurs (liftoff of the second stride).

Fig. 7. Leg forces for the SLIP-embedding controller (left), and the rigid target model controller (right), and for the first four steps of Fig. 6. Upper plots show total leg forces (solid) and spring forces (dashed); bottom plots show leg actuator forces computed by (76). 
TABLE II

CONTROL EFFORT: SLIP-EMBEDDING AND RTM CONTROLLERS

\begin{tabular}{|c|c|c|c|c|}
\hline Perturbation & Control & Stride & $\left(u_{1}^{a}, u_{2}\right)^{\max }$ & $\left(W_{1}, W_{2}\right)^{\text {total }}$ \\
\hline \hline$\delta \theta=+4 \mathrm{deg}$ & SLIP & 4 & $(54,28)$ & $(24,18)$ \\
\cline { 2 - 5 } & RTM & 6 & $(442,15)$ & $(71,24)$ \\
\hline$\delta \theta=-3 \mathrm{deg}$ & SLIP & 4 & $(50,26)$ & $(16,19)$ \\
\cline { 2 - 5 } & RTM & 4 & $(382,21)$ & $(55,19)$ \\
\hline \hline$\delta \dot{x}_{\mathrm{c}}=+0.9 \frac{m}{\mathrm{~s}}$ & SLIP & 6 & $(418,64)$ & $(110,40)$ \\
\cline { 2 - 5 } & RTM & 12 & $(448,37)$ & $(242,76)$ \\
\hline$\delta \dot{x}_{\mathrm{c}}=-1.4 \frac{\mathrm{m}}{\mathrm{s}}$ & SLIP & \multicolumn{3}{|c|}{ Outside of the basin of attraction } \\
\cline { 2 - 5 } & RTM & 15 & $(486,15)$ & $(236,47)$ \\
\hline
\end{tabular}

\section{Qualitative Discussion}

The significantly lower leg actuator forces reported for the SLIP-embedding controller in Table II are due to the fact that, in this case, the control input acts in concert with the spring. To be precise, as was mentioned in Remark 8 , the intuitive meaning of the feedback law given by (58) is that the ASLIP (actual) leg force, $u_{1}$, is rendered equal to the projection of the SLIP (virtual) leg force, $F_{\mathrm{ES}-\mathrm{SLIP}}$, along the direction of the actual leg. In view of (76), to achieve this prescription the leg actuator $u_{1}^{\mathrm{a}}$ is only required to "shape" the actual spring force $k_{\mathrm{A}}\left(l_{\text {nat }}-l\right)$, so that the required central spring force, $F_{\mathrm{ES}-\mathrm{SLIP}}$, along the virtual (SLIP) leg direction is developed. As can be seen in Fig. 1, for physically reasonable torso pitch angles, the angle between the actual leg and the virtual leg direction is small. Consequently, small actuator effort suffice to "shape" the spring force of the actual leg to achieve this projection.

Concerning the lower power required by the SLIPembedding controller, this is attributed to the fact that much of the work done on the leg is provided by the spring. Hence, in decelerating the COM during the compression part of the stance phase, only a small amount of energy is dissipated in the leg actuator. Finally, another particularly important advantage of the SLIP-embedding controller is that, under reasonable conditions, it does not violate the ground contact constraints. In contrast, the rigid target model controller frequently commands leg forces that violate the unilateral constraints characterizing the toe/ground interaction. For instance, this occurs when the current leg length exceeds the commanded value. On such occasions, the controller attempts to shorten the leg by "pulling" the ground, often resulting in forces that violate the unilateral ground constraint.

These results demonstrate the significance of designing the HZD of running to respect the compliance available in the open-loop system. Otherwise, the beneficial effects of the actual leg spring may be canceled by the control inputs during transients.

\section{COnClusion}

In this paper, a framework for the systematic design of control laws with provable properties for the ASLIP, an extension of the SLIP that includes nontrivial torso pitch dynamics, is proposed. The ASLIP can be envisioned as a "building block" toward the construction of controllers for more elaborate models that constitute more accurate representations of legged robots. The control law proposed acts on two levels. On the first level, continuous in-stride control asymptotically stabilizes the torso pitch, and creates an invariant surface on which the closed-loop dynamics is diffeomorphic to a target compliant system -in this particular case, the SLIP dynamics. On the second level, an event-based controller is used to stabilize the target compliant system along a desired periodic orbit. An immediate practical consequence of this method for the ASLIP is that it affords the direct use of a large body of controller results that are available in the literature for the SLIP. Furthermore, it is deduced through comparisons of the SLIP-embedding controller with a rigid target model controller creating a one-degree-of-freedom non-compliant subsystem, that the underlying compliant nature of the SLIP enhances performance by significantly improving the transient response and reducing actuator effort. This paper should be viewed as a first step toward a general framework of controller design exhibiting compliant hybrid zero dynamics.

\section{APPENDIX}

In this appendix, the formulas for the stance-to-flight and flight-to-stance transition maps of the ASLIP are presented. All the transition maps correspond to coordinate transformations taking stance to flight and flight to stance coordinates.

\section{A. ASLIP stance-to-flight transition maps}

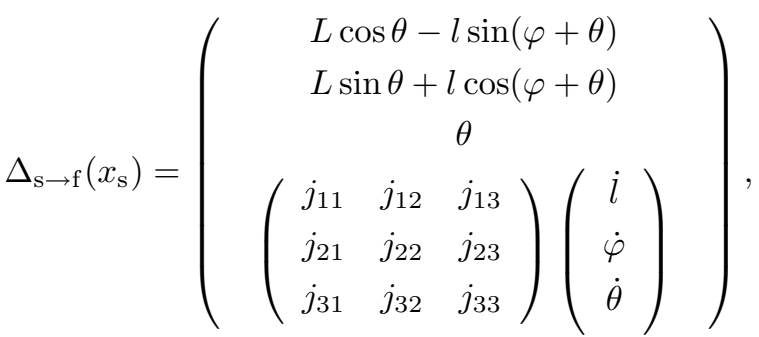

where

$$
\begin{gathered}
j_{11}=-\sin (\varphi+\theta), j_{12}=-l \cos (\varphi+\theta), \\
j_{13}=-l \cos (\varphi+\theta)-L \sin \theta, \\
j_{21}=\cos (\varphi+\theta), j_{22}=-l \sin (\varphi+\theta), \\
j_{23}=-l \sin (\varphi+\theta)+L \cos \theta, \\
j_{31}=0, j_{32}=0, j_{33}=1 .
\end{gathered}
$$

\section{B. ASLIP flight-to-stance transition map}

$$
\Delta_{\mathrm{f} \rightarrow \mathrm{s}}\left(x_{\mathrm{f}}, \alpha_{\mathrm{f}}\right)=\left(\begin{array}{c}
\sqrt{\left(L \cos \theta-x_{\mathrm{c}}\right)^{2}+\left(L \sin \theta-y_{\mathrm{c}}\right)^{2}} \\
\arctan \left(\frac{L \cos \theta-x_{\mathrm{c}}}{y_{\mathrm{c}}-L \sin \theta}\right)-\theta \\
\theta \\
\left(\begin{array}{ccc}
j_{11}^{-1} & j_{12}^{-1} & j_{13}^{-1} \\
j_{21}^{-1} & j_{22}^{-1} & j_{23}^{-1} \\
j_{31}^{-1} & j_{32}^{-1} & j_{33}^{-1}
\end{array}\right)\left(\begin{array}{c}
\dot{x}_{\mathrm{c}} \\
\dot{y}_{\mathrm{c}} \\
\dot{\theta}
\end{array}\right)
\end{array}\right)
$$


where

$$
\begin{aligned}
j_{11}^{-1}= & \frac{x_{\mathrm{c}}-L \cos \theta}{A\left(x_{\mathrm{c}}, y_{\mathrm{c}}, \theta\right)}, j_{12}^{-1}=\frac{y_{\mathrm{c}}-L \sin \theta}{A\left(x_{\mathrm{c}}, y_{\mathrm{c}}, \theta\right)}, \\
& j_{13}^{-1}=\frac{L x_{\mathrm{c}} \sin \theta-L y_{\mathrm{c}} \cos \theta}{A\left(x_{\mathrm{c}}, y_{\mathrm{c}}, \theta\right)}, \\
j_{21}^{-1}= & \frac{L \sin \theta-y_{\mathrm{c}}}{A^{2}\left(x_{\mathrm{c}}, y_{\mathrm{c}}, \theta\right)}, j_{22}^{-1}=\frac{x_{\mathrm{c}}-L \cos \theta}{A^{2}\left(x_{\mathrm{c}}, y_{\mathrm{c}}, \theta\right)}, \\
j_{23}^{-1}= & \frac{x_{\mathrm{c}}\left(L \cos \theta-x_{\mathrm{c}}\right)+y_{\mathrm{c}}\left(L \sin \theta-y_{\mathrm{c}}\right)}{A^{2}\left(x_{\mathrm{c}}, y_{\mathrm{c}}, \theta\right)}, \\
& j_{31}^{-1}=0, j_{32}^{-1}=0, j_{33}^{-1}=1,
\end{aligned}
$$

with

$$
A\left(x_{\mathrm{c}}, y_{\mathrm{c}}, \theta\right)=\sqrt{\left(L \cos \theta-x_{\mathrm{c}}\right)^{2}+\left(L \sin \theta-y_{\mathrm{c}}\right)^{2}} .
$$

\section{ACKNOWLEDGMENT}

The authors would like to thank Dr. J. W. Hurst for providing the mechanical drawing appearing in Fig. 1, which corresponds to a leg of robotic testbeds constructed in a collaborative effort between the University of Michigan and Carnegie Mellon University.

\section{REFERENCES}

[1] M. Ahmadi and M. Buehler, "Stable control of a simulated one-legged running robot with hip and leg compliance," IEEE Transactions on Robotics and Automation, vol. 13, no. 1, pp. 96-104, Feb. 1997.

[2] — "Control passive dynamic running experiment with the ARL Monopod II," IEEE Transactions on Robotics, vol. 22, no. 5, pp. 974 986, Oct. 2006

[3] R. Altendorfer, D. E. Koditschek, and P. Holmes, "Stability analysis of legged locomotion models by symmetry-factored return maps," International Journal of Robotics Research, vol. 23, no. 10-11, pp. 979-999, Oct. 2004.

[4] A. D. Ames and R. D. Gregg, "Stably extending two-dimensional bipedal walking to three," in Proceedings of the American Control Conference, New York, U.S.A., July 2007, pp. 2848-2854.

[5] A. D. Ames, R. D. Gregg, E. D. B. Wendel, and S. Sastry, "On the geometric reduction of controlled three-dimensional bipedal robotic walkers," in Proceedings of the 3rd IFAC Wrokshop on Lagrangian and Hamiltonian Methods for Nonlinear Control, Nagoya, Japan, July 2006.

[6] R. Blickhan, "The spring-mass model for running and hopping," Journal of Biomechanics, vol. 22, no. 11-12, pp. 1217-1227, 1989.

[7] R. Blickhan and R. J. Full, "Similarity in multi-legged locomotion: bouncing like a monopode," Journal of Comparative Physiology A, vol. 173, pp. 509-517, 1993

[8] W. M. Boothby, An Introduction to Differentiable Manifolds and Riemannian Geometry, 2nd ed. New York: Academic Press, 1975.

[9] M. Bühler, D. Koditschek, and P. Kindlmann, "A family of robot control strategies for intermittent dynamics environments," IEEE Control Systems Magazine, vol. 10, no. 2, pp. 16-22, Feb. 1990.

[10] N. Cherouvim and E. Papadopoulos, "Single actuator control analysis of a planar 3DOF hopping robot," in Robotics: Science and Systems I, S. Thrun, G. Sukhatme, and S. Schaal, Eds. MIT Press, 2005, pp. $145-152$.

[11] C. Chevallereau, G. Abba, Y. Aoustin, F. Plestan, E. R. Westervelt, C. Canudas, and J. W. Grizzle, "RABBIT: a testbed for advanced control theory," IEEE Control Systems Magazine, vol. 23, no. 5, pp. 57-79, Oct. 2003.

[12] C. Chevallereau, E. R. Westervelt, and J. W. Grizzle, "Asymptotically stable running for a five-link, four-actuator, planar bipedal robot," International Journal of Robotics Research, vol. 24, no. 6, pp. 431464, June 2005.

[13] C. François and C. Samson, "Running with constant energy," in Proceedings of the IEEE International Conference on Robotics and Automation, vol. 1, San Diego, U.S.A., May 1994, pp. 131-136.
[14] - "A new approach to the control of the planar one-legged hopper," International Journal of Robotics Research, vol. 17, no. 11, pp. 11501166, Jan. 1998.

[15] R. Full and D. E. Koditschek, "Templates and anchors: Neuromechanical hypotheses of legged locomotion on land," Journal of Experimental Biology, vol. 202, pp. 3325-3332, Dec. 1999.

[16] R. M. Ghigliazza, R. Altendorfer, P. Holmes, and D. E. Koditschek, "A simply stabilized running model," SIAM Journal of Applied Dynamical Systems, vol. 2, no. 2, pp. 187-218, May 2003.

[17] P. Gregorio, M. Ahmadi, and M. Buehler, "Design, control, and energetics of an electrically actuated legged robot," IEEE Transactions on Systems, Man and Cybernetics, vol. 27, no. 4, pp. 626-634, Aug. 1997.

[18] J. W. Grizzle, "Papers on biped robots," http://www.eecs.umich.edu/ $\sim$ grizzle/papers/robotics.html, retrieved on 23 June 2008.

[19] J. W. Grizzle, G. Abba, and F. Plestan, "Asymptotically stable walking for biped robots: Analysis via systems with impulse effects," IEEE Transactions on Automatic Control, vol. 46, no. 1, pp. 51-64, Jan. 2001.

[20] P. Holmes, R. J. Full, D. Koditschek, and J. Guckenheimer, "The dynamics of legged locomotion: Models, analyses, and challenges," SIAM Review, vol. 48, no. 2, pp. 207-304, May 2006.

[21] J. W. Hurst, J. E. Chestnutt, and A. A. Rizzi, "Design and philosophy of the BiMASC, a higly dynamic biped," in Proceedings of the IEEE International Conference of Robotics and Automation, Roma, Italy, Apr. 2007, pp. 1863-1868.

[22] S. H. Hyon and T. Mita, "Development of a biologically inspired hopping robot - "Kenken"," in Proceedings of the IEEE International Conference of Robotics and Automation, Washington DC, U.S.A., 2002, pp. 3984-3991.

[23] S.-H. Hyon and T. Emura, "Symmetric walking control: Invariance and global stability," in Proceedings of the IEEE International Conference on Robotics and Automation, Barcelona, Spain, 2005, pp. 1455-1462.

[24] — "Energy-preserving control of a passive one-legged running robot," Advanced Robotics, vol. 18, no. 4, pp. 357-381, May 2004.

[25] A. Isidori, Nonlinear Control Systems, 3rd ed. Berlin: Springer-Verlag, 1995.

[26] H. K. Khalil, Nonlinear Systems, 3rd ed. Upper Saddle River, NJ: Prentice Hall, 2002.

[27] D. E. Koditschek and M. Bühler, "Analysis of a simplified hopping robot," International Journal of Robotics Research, vol. 10, no. 6, pp. 587-605, Dec. 1991.

[28] T. McGeer, "Passive dynamic walking," International Journal of Robotics Research, vol. 9, no. 2, pp. 62-82, Apr. 1990.

[29] R. M'Closkey and J. Burdick, "Periodic motions of a hopping robot with vertical and forward motion," International Journal of Robotics Research, vol. 12, no. 3, pp. 197-218, June 1993.

[30] K. D. Mombaur, R. W. Longman, H. G. Bock, and J. P. Schlöder, "Stable one-legged hopping without feedback and with a point foot," in Proceeding of the IEEE International Conference on Robotic and Authomation, Washington DC, U.S.A., May 2002, pp. 3978-3983.

[31] B. Morris and J. W. Grizzle, "A restricted Poincaré map for determining exponentially stable periodic orbits in systems with impulse effects: Application to bipedal robots," in Proceedings of the IEEE International Conference on Decision and Control, Seville, Spain, Dec. 2005, pp. 4199-4206.

[32] — "Hybrid invariance in bipedal robots with series compliant actuators," in Proceedings of the IEEE International Conference on Decision and Control, San Diego, USA, Dec. 2006, pp. 4793-4800.

[33] J. Nakanishi, T. Fukuda, and D. E. Koditschek, "A brachiating robot controller," IEEE Transactions on Robotics and Automation, vol. 16, no. 2, pp. 109-123, Apr. 2000.

[34] I. Poulakakis and J. W. Grizzle, "Formal embedding of the spring loaded inverted pendulum in an asymmetric hopper," in Proceedings of the European Control Conference, Kos, Greece, July 2007.

[35] — "Monopedal running control: SLIP embedding and virtual constraint controllers," in Proceedings of the IEEE/RSJ International Conference of Intelligent Robots and Systems, San Diego, U.S.A., Oct. 2007.

[36] M. H. Raibert, Legged Robots that Balance. Cambridge, MA: MIT Press, 1986.

[37] U. Saranli and D. Koditschek, "Template based control of hexapedal running," in Proceecings of the IEEE International Conference on Robotics and Automation, vol. 1, Taipei, Taiwan, Sept. 2003, pp. 13741379 .

[38] U. Saranli, W. Schwind, and D. E. Koditschek, "Toward the control of a multi-jointed, monoped runner," in Proceedings of the IEEE International Conference on Robotics and Automation, vol. 3, Leuven, Belgium, May 1998, pp. 2676-2682. 
[39] W. J. Schwind, "Spring loaded inverted pendulum running: A plant model," Ph.D. dissertation, University of Michigan, 1998.

[40] W. J. Schwind and D. E. Koditschek, "Control of forward velocity for a simplified planar hopping robot," in Proceedings of the IEEE Inernational Conference of Robotics and Automation, vol. 1, Nagoya, Japan, May 1995, pp. 691-696.

[41] J. E. Seipel and P. Holmes, "Running in three dimensions: Analysis of a point-mass sprung-leg model," International Journal of Robotics Research, vol. 24, no. 8, pp. 657-674, Aug. 2005.

[42] A. Seyfarth, H. Geyer, M. Gunther, and R. Blickhan, "A movement criterion for running," Journal of Biomechanics, vol. 35, no. 5, pp. 64955, May 2002.

[43] A. Seyfarth, H. Geyer, and H. Herr, "Swing leg retraction: A simple control model for stable running," Journal of Experimental Biology, vol. 206, pp. 2547-2555, 2003.

[44] M. W. Spong, S. Hutchinson, and M. Vidyasagar, Robot Modeling and Control. New York: John Wiley \& Sons, 2006.

[45] M. Spong and F. Bullo, "Controlled symetries and passive walking," IEEE Transactions on Automatic Control, vol. 50, no. 7, pp. 1025-1031, July 2003.

[46] A. Vakakis, J. Burdick, and T. Caughey, "An "interesting" strange attractor in the dynamics of a hopping robot," International Journal of Robotics Research, vol. 10, no. 6, pp. 606-618, Dec. 1991.

[47] E. R. Westervelt, J. W. Grizzle, C. Chevallereau, J. H. Choi, and B. Morris, Feedback Control of Dynamic Bipedal Robot Locomotion. Taylor \& Francis/CRC Press, 2007.

[48] E. R. Westervelt, J. W. Grizzle, and D. E. Koditschek, "Hybrid zero dynamics of planar biped walkers," IEEE Transactions on Automatic Control, vol. 48, no. 1, pp. 42-56, Jan. 2003. 